\title{
UN TRASFORMATORE DINAMICO PER CORRENTI ALTERNATE.
}

\author{
Nota di A. G. ROSSI.
}

(Continuazione e fine).

o 9 bis. - Autotrasformatore in derivazione $e$ in serie.

A complemento del $\S 9$, aggiungasi quanto segue.

Lo schema dell' inserzione descritto in fig. 15, pag. [43] può dirsi dell'autotrasformatore in derivazione, per distinguerlo da un altro, effettuabile secondo Ia fig. 15 bis., che diremo dell'autotrasformatore in serie. Nel primo, uno degli avvolgimenti è in derivazione sulla linea e l'altro è in serie. Nel secondo, entrambi gli avvolgimenti sono in serie, l' uno sul filo di andata e l'altro sul filo di ritorno della linea.

Nei due casi, occorre all'atto pratico tener conto dei sensi relativi delle correnti nei due avvolgimenti.

Le equazioni (1) e conseguenti del $\S 9$ rappresentano le cose genericamente, senza riguardo alle due possibilità d'inserzione, corrispondenti ai due versi della corrente in una delle spirali rispetto all'altra: $s$ ' intende che il termine dell' induzione dinamica cambia di segno se si capovolge l'una delle spirali. Dette equazioni vanno lette :

$$
\mathbf{V}=\mathbf{J}_{\mathbf{1}} \mathbf{Z}_{1} \mp j \mathrm{~K} \mathbf{Y}^{\prime} \mathbf{J}_{e}=\mathbf{J}_{\theta} \mathbf{Z}_{e} \mp j \mathrm{~K} \mathbf{Y}^{\prime} \mathbf{J}_{\mathbf{1}} \text {. }
$$

Il segno inferiore corrisponde alle connessioni indicate dalla fig. 15 e fornisce i risultati voluti, cioè la tensione secondaria $\mathrm{E}_{2}$, l'ammettenza complessiva $y^{*}$, i rapporti di trasformazione, ete. funzioni crescenti della velocità $\mathrm{K}$.

Nella espressione simbolica della ammettenza complessiva

$$
\mathbf{Y}^{*}=\frac{\mathbf{Z}_{e}+2\left(\mathbf{Z}_{1}^{\prime}-j \mathbf{K} \mathbf{Y}^{\prime}\right)}{\mathbf{Z}_{1}^{\prime}\left(\mathbf{Z}_{1}^{\prime}+\mathbf{Z}_{e}\right)+K^{3} \mathbf{Y}^{\prime 2}}=\frac{\mathbf{Y}_{1}^{\prime}+\mathbf{Y}_{2}^{\prime}-2 j \mathbf{K} \mathbf{Y}^{\prime} \mathbf{Y}_{1}^{\prime} \mathbf{Y}_{3}^{\prime}}{1+\mathbf{K}^{3} \mathbf{Y}^{\prime 3} \mathbf{Y}_{1}^{\prime} \mathbf{Y}_{2}^{\prime}}
$$

il modulo del numeratore è funzione di $\mathrm{K}$ sempre crescente e il modulo del denominatore discende dall'origine verso un 
minimo, come quello della impedenza primaria, ciò che conduce $y^{*}$ a variare secondo curre analoghe a quelle della fig. 16.

Questa osservazione ovvia, riguardante il segno del fattore d' induzione $K \mathrm{Y}^{\prime}$ per il caso dello sehema in fig. 15, nulla cambia pero alla discussione generica fatta sull' autotrasformatore in derivazione e ne lascia intatte le conclusioni.

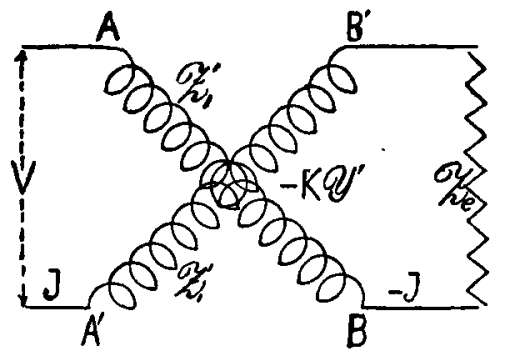

Fig. 15 (bis).

Interesse offre anche l'autotrasformatore in serie (fig. 15 bis), inserzione analoga a quella proposta dal Kelly per una generatrice asincrona (i tre avvolgimenti dello statore trifase separatamente inseriti sulla linea fra il generatore sincrono e gli apparecchi utilizzatori), la quale, trascinata oltre sincronismo da una motrice, genera sulla linea una componente attiva della f. e. $\mathrm{m}$. assorbendone però una componente reattiva. Nel nostro caso, il funzionamento è molto più semplice; a rotore in moto, con qualunque velocità, i due avvolgimenti sono sedi di forze elettromotrici dinamiche della forma $\mathbf{K} \mathbf{Y}^{\prime}$, le componenti delle quali si aggiungono o si sottraggono, a seconda delle connessioni, alle componenti delle cadute di tensione sull' impedenza di linea, percorsa dalla corrente I.

L'equazione del circuito è semplicemente

$$
\mathbf{V}=\mathbf{J}\left[\mathbf{Z}_{\boldsymbol{\theta}}+\mathbf{2}\left(\mathbf{Z}_{\mathbf{1}}^{\prime} \mp j \mathbf{K} \mathbf{Y}^{\prime}\right)\right]=\mathbf{J} \mathbf{Z}_{*} .
$$


Ool segno inferiore, che corrisponde alle connessioni indicate in fig. 15 bis, il modulo della impedenza complessiva

$$
\mathbf{Z}_{*}=r_{e}+2\left(r_{1}^{\prime}-\mathrm{K} b^{\prime}\right)-j\left[\lambda_{e}+2\left(\lambda_{1}{ }^{\prime}-\mathrm{K} g^{\prime}\right)\right],
$$

è all'origine una funzione decrescente della velocita $K$ :

$$
z^{*}=\sqrt{z_{1 e}{ }^{2}-4 y^{1 /} \mathrm{K}\left[\lambda^{\prime} r_{1 e}+r^{1} \lambda_{1 e}-\mathbf{K}\right]},
$$

la quale, partendo da $z_{1 e}=\sqrt{\left(r_{e}+2\right.} \overline{\left.r_{1}^{\prime}\right)^{2}+\left(\lambda_{e}+2 \lambda_{1}^{\prime}\right)^{2}}$, col gradiente $-2 y_{1 e}\left(b^{\prime} r_{1 e}+g^{\prime} \lambda_{1 e}\right)$, discende ad un minimo $z_{*} \min =$ $=y^{\prime}\left(\lambda^{\prime} \lambda_{1 e}-r^{\prime} r_{1 e}\right)$, con la velocita $K^{\prime}=\left(r^{\prime} \lambda_{1 e}+\lambda^{\prime} r_{1 e}\right) / 2$, per per poi risalire indefinitamente, ripassando per il valore iniziale $z_{\text {se }}$ con la velocità $2 \mathrm{~K}^{\prime}$. Il minimo è tanto più basso, per qualsiasi $r_{e} \cdot j \lambda_{e}$, quanto più prossimamente l'apparecchio soddisfi alla condizione limite $\left(21^{*}\right)$. Ete.

Però, alla semplicità di questa disposizione, con la quale il T. D. lavorerebbe a guisa di survoltrice asincrona nel modo e nel senso più generale, corrisponde una mediocre utilizzazione delle proprietà dell'apparecchio; non solo perchè gli avvolgimenti di statore vengono eccitati da una piccola parte della totale tensione di linea, ma soprattuto perchè le componenti dinamiche sottrattive $\mathbf{K} b^{\prime}$ e $\mathbf{K} g^{\prime}$, che dipendono solo dal rotore, non possono, a velocità limitate, raggiungere notevoli valori. Invece, quando l' apparecchio funztona veramente in condizioni di trasformatore, la impedenza dinamica dell'avvolgimento è : $\mathbf{Z}_{1}^{\prime}+\mathrm{K}^{\mathbf{8}} \mathbf{Y}^{\prime \mathbf{s}}\left(\mathbf{Y}_{1}^{\prime}+\mathbf{Y}_{e}\right)$, e le componenti dinamiche dell' impedenza, oltre che dal quadrato della velocità, dipendono anche dal circuito di statore.

\section{\$11. - Induzione mutua costante nei circuiti degli statori.}

a) Nella trattazione del T. D. si è supposto che fra stàtore primario e statore secondario non esistesse induzione mutua diretta, nè in assenza nè in presenza del $D$ rotore in quiete. Questo è invero un caso puramente teorico, poichè è generalmente impossibile costruire i due avvolgimenti, o su due statori distinti o sopra un unico statore, nelle condizioni di identita supposte dalla teoria e fra loro rigorosamente 
spostati di un quarto di passo. All' atto pratico, si ritrova sempre una mutua induzione diretta attraverso il $D$ rotore. Tale difetto costruttivo tende a sparire, per via di compensazioni, con l'aumentare del numero delle coppie di poli. Osservazioni sperimentali offerte dal funzionamento di un tipo della macchina costrutta dall'a., il quale comprende due statori equimultipolari separati, spostabili angolarmente l'uno rispetto all'altro e affacciati sulle due fronti del $D$. rotore, suggerirono lo studio analitico del caso più generale che i due statori stessi presentino attraverso il $\mathrm{D}$. in quiete una mutua induzione diversa da zero, supponendo che essa provenga da ciò che $i$ due sistemi di spirali fisse sieno spostati fra loro di un intervallo angolare differente da un quarto dỉ passo. Tale trattazione ') fu fatta adottando, per definire la sregolazione dell'apparechio, un coefficiente numerico $x$, variabile fra +1 e -1 , come potrebbe essere il cos di un angolo misurato su una circonferenza di luughezza uguale at passo dell'avvolgimento; questa ipotesi semplicista si trovò sufficientemente giustificata dalla concordanza fra le deduzioni e le osservazioni sperimentali. Riassumiamo qui brevemente i risultati di questo studio.

Il fatore d'induzione complesso fra $i$ due statori acquista la forma: $\mu(\gamma+j \beta)=$

$$
\mu\left[k u(1-x) g^{\prime}-\mu x\left(b+b^{\prime}\right)+j\left(k u(1-x) b^{\prime}+\mu x\left(g+g^{\prime}\right)\right)\right] \text {. }
$$

La corrente secondaria è diversa da zero per $u=0$, cambia di segno e svanisce insieme ad $x$. Per $u \gtrless 0$, a seconda dei segni relativi di $k u(1-x)$ e di $\mu x$, l' una delle componenti del fattore d'induzione può essere inizialmente crescente - l'altra decrescente, col crescere della velocita in valore assoluto: il funzionamento viene dunque a diperidere ora anche dal verso della velocità impressa al rotore.

1) Atti della R. Accademia delle Scienze, di Torino., Vol, 51; 19 Marzo. 1916. 
Per ciò che riguarda le componenti della impedenza apparente, in un campo di variazioni di $x$ lontano dal suo valor massimo 1 , per ogui dato valore della anomalia $x$ esistono valori di $u$ che annullano la resistenza apparente o la reattanza apparente, riproducendo le vicissitudini del caso normale; e per ciascuna velocità $u$, esistono valori dell'anomalia $x$ che portano agli stessi eventi.

La tensione secondaria a vuoto $\left(\mathrm{E}_{8}\right)$ con $\mathrm{V}_{1}$ costante acquista, in funzione della velocità, un andamento parabolico, partendo da un valore iniziale $>0$, con gradiente $\gtrless 0$ a seconda lel verso della velocità $u$, ma sempre inferiore in valore assoluto al gradiente $\operatorname{tg} \varepsilon$ della retta che si ba nel caso normale. Corrispondentemente, la corrente secondaria inizialmente è crescente oppure decrescente. Nel secondo caso, $I_{2}$ raggiunge un minimo, dopo di che torna a crescere avviandosi al suo massimo, il quale non è che ritardato; nel primo caso invece, il suo massimo è avanzato, cioè si raggiunge con una velocità minore.

In conclusione, finchè $\mathrm{i}$ termini dipendenti da $x$ non superino l'importanza di termini di correzione, i caratteri generali del funzionamento dell'apparecchio rimangono praticamente inalterati. Per valori sensibili o finiti della sregolazione $x$, la sua influenza si fa sentire nel campo delle velocità più basse, per avanzare oppure per ritardare l'aumento iniziale delle correnti, a seconda del verso della velocità. Quanto maggiore sia la sregolazione $x$ e tanto più alta diviene la velocità alla quale scompare la sua influenza sul valore delle correnti, specialmente della secondaria.

b) Presenta interesse generale il caso che esista esternamente nel circuito dei due statori, nelle condizioni normali, una mutua induzione costante fra i due circuiti primario e secondario del T. D., procurata da un ordinario trasformatore statico (T. S.). 
I due circuiti del T. D. sono, in normali condizioni, fra loro isolati elettromagneticamente, finchè il rotore è in quiete. Per le applicazioni dell' apparecchio, può occorrere di associarlo ad un T.S., affinchè passi energia elettrica dalla linea primaria a quella secondaria anche a rotore fermo, e la rotazione di quest'ultimo intarvenga per iniettare nuova energia nella linea secondaria. Disposizioni per questo scopo furono già studiate con l'inserzione del T. D. come autotrasformatore.

Un T. D. e un T. S. possono ora venire associati o in serie, fig. 18, oppure in derivazione (parallelo), fig. 19. $\mathrm{Nel}$ primo caso, sulla linea secondaria si sommano le tensioni, nel secondo caso si sommano le correnti generate dai due apparecchi.

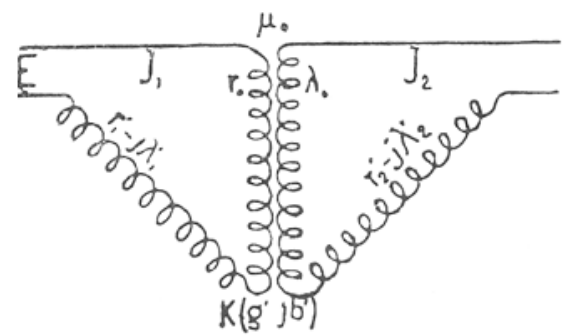

Fig. 18.

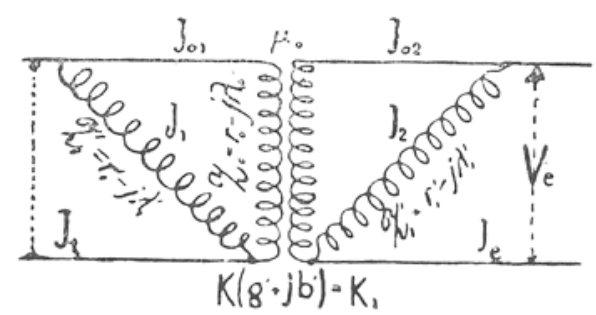

Fig. 19.

Per conservare simmetria (e semplicita di trattazione), supporremo che il T.S. consista in due eguali avvolgimenti, di resistenza $r_{0}$ e reattanza $\lambda_{0}$, fra $\mathrm{i}$ quali regni il « fattoro $d^{\prime}$ induzione $\gg \mu_{0}$ costante. 
Se il primario e il secondario $\left(r_{0}, \lambda_{0}, \mu_{0}\right)$ sieno rispettivamente in serie nei due circuiti corrispondenti del T. D., le equazioni del sistema sono:

$$
\begin{aligned}
& \mathbf{V}_{1}=\mathbf{J}_{1}\left(\mathbf{Z}_{0}+\mathbf{Z}_{1}^{\prime}\right)-j \mathbf{J}_{2}\left(\mu_{0}+\mathbf{K} \mathbf{Y}^{\prime}\right) \\
& 0=\mathbf{J}_{2}^{\prime}\left(\mathbf{Z}_{0}+\mathbf{Z}_{2}{ }^{\prime}\right)-j \mathbf{J}_{1}\left(\mu_{0}+\mathbf{K} \mathbf{Y}^{\prime}\right),
\end{aligned}
$$

ove $\mathbf{Z}_{2}^{\prime}=\mathbf{Z}_{1}^{\prime}+\mathbf{Z}_{e}$. Il rapporto di trasformazione delle correnti è

$$
\frac{J_{2}}{J_{1}}=j \frac{\mu_{0}+K Y^{\prime}}{Z_{0}+Z_{2}^{\prime}} \quad, L \frac{\pi}{2}+\operatorname{arctg} \frac{\lambda_{0}+\lambda_{2}{ }^{\prime}}{r_{0}+r_{2}{ }^{\prime}}+\operatorname{arctg} \frac{K b^{\prime}}{\mu_{0}+K g^{\prime}} .
$$

e il rapporto di trasformazione delle tensioni, con $\mathrm{J}_{2}=0$,

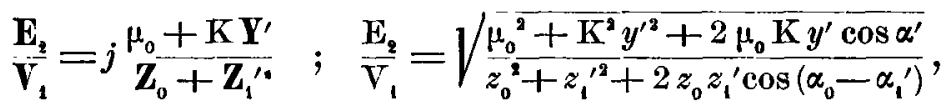

funzione che può essere continuamente crescente, il fattore $\mu_{0} \mathrm{~K}$ essendo $\geqq 0$ a seconda del senso della inserzione o della velocità del rotore. $L$ 'impedenza $\left(\mathbf{Z}_{0}+\mathbf{Z}_{\mathbf{1}}^{\prime}\right)$ può venir diminuita, se si derivi un condensatore opportuno su $z_{0}$.

Abbreviando con:

$$
z_{02}{ }^{2}=\left(r_{0}+r_{3}{ }^{\prime 2}+\left(\lambda_{0}+\lambda_{2}\right)^{2} \quad ; \quad g_{02}{ }^{\prime}=\left(r_{0}+r_{2}{ }^{\prime}\right) / z_{02}{ }^{2}\right.
$$

$b_{02}=\left(\lambda_{0}+\lambda_{2}{ }^{\prime}\right) / z_{02}{ }^{2}$, e similmente con $z_{01}{ }^{2}=r_{02}{ }^{2}+\lambda_{01}{ }^{2}$, etc.,

le componenti della impedenza opposta alla tensione primaria,

$$
\mathrm{Z}=\mathrm{R}-j \Lambda=\mathbf{V}_{1} \mid \mathbf{J}_{1},
$$

possono scriversi :

$$
\begin{aligned}
\mathbf{R} & =r_{0}+r_{1}^{\prime}+g_{02}\left[\left(\mu_{0}+\mathrm{K} g^{\prime}\right)^{2}-K^{2} b^{\prime 2}\right]-b_{02}\left[2 \mathrm{~K} b^{\prime}\left(\mu_{0}+\mathrm{K} g^{\prime}\right)\right] \\
& =r_{01}+\mu_{0}^{2} g_{02}+K^{2}\left[g_{02}\left(g^{\prime 2}-b^{\prime 3}\right)-b_{02} 2 b^{\prime} g^{\prime}\right]+2 \mu_{0} \mathrm{~K}\left(g^{\prime} g_{02}-b^{\prime} b_{02}\right) \\
\Lambda & =\lambda_{0}+\lambda_{1}{ }^{\prime}-b_{02}\left[\left(\mu_{0}+K g^{\prime}\right)^{2}-K^{2} b^{\prime 2}\right]-g_{02}\left[2 \mathrm{~K} b^{\prime}\left(\mu_{0}+\mathrm{K} g^{\prime}\right)\right] \\
& =\lambda_{01}-\mu_{0}^{2} b_{02}-K^{2}\left[b_{02}\left(g^{\prime 2}-b^{\prime 3}\right)+g_{02} 2 b^{\prime} g^{\prime}\right]-2 \mu_{0} \mathrm{~K}\left(g^{\prime} b_{02}+b^{\prime} g_{02}\right)
\end{aligned}
$$

Sono funzioni paraboliche di $K$, crescenti o decrescenti a seconda dei segni dei coefficienti di $K^{2}$ e $K$, dipendenti dal circuito secondario; $\mu_{0} K$ ha un segno variabile ad arbitrio; da quest'ultimo termine dipende il gradiente iniziale; etc. Un maggior numero di elementi influisce dunque sul- 
l'andamento dell' impedenza funzione della velocitè, in confronto al caso del T. D. semplice; ma sempre essa è caratterizzata dall'esistenza di un minimo, a cui consegue un massimo della corrente secondaria, il quale, per effetto del T.S., si può raggiungere con velocità minori e con un gradiente medio aumentato. Questa disposizione offre vantaggi per l'impiego del relais telefonico.

c) Merita un più esteso esame il caso che i due primari e i due secondari sieno rispettivamente congiunti in parallelo (fig. 19).

L' importanza di questo caso si può prevedere, se si ammette che la funzione principale del trasformatore dinamico nel circuito primario sia quella di avanzare la fase della corrente sulla tensione applicata, creando, col crescere della velocità, prima una componente reattiva poi una componente attiva, disponibili per il circuito primario esterno. Tali componenti possono ora venire assorbite dal primario del trasformatore statico che gli è accoppiato.

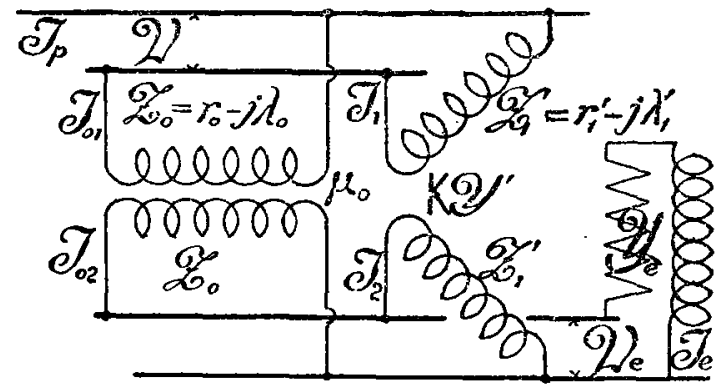

Fig. 19 (bis).

Se, in particolare, il primario del T. D. fornisse, ad una certa velocita, al primario del T. S. tutta la corrente oziosa di cui esso abbisogna, si avrebbe una specie di effetto di risonanza nel « circuito » dei due primari, quale si potrebbe ottenere con un condensatore di capacità opportuna in pa- 
rallelo sul primario $\left(r_{0}, \lambda_{0} ; \mu_{0}\right)$. La trattazione seguente è diretta a formulare questa previsione.

\section{sं 12. - Un T. D. in parallelo con un T. S.}

Sieno dunque: $J_{01}$ e $J_{0,}$ le correnti negli avvolgimenti del T. S., $d$ ' impedenza originaria $Z_{0}=r_{0}-j \lambda_{0} ; J_{1} \otimes J_{2}$ le correnti negli avvolgimenti del T. D., d'impedenza originaria $\mathbf{Z}_{1}^{\prime}=r_{1}^{\prime}-j \lambda_{1}{ }^{\prime} ; \mathbf{J}_{p}$ la corrente totale primaria, che porta la tensione $\mathbf{V}$ alla coppia degli avvolgimenti primari derivati; $\boldsymbol{J}_{e}$ la corrente nel circuito secondario esterno, o di linea, d'impedenza $\mathbf{Z}_{e}=r_{e}-j \lambda_{e} ; \mathbf{V}_{e}$ la tensione ai nodi secondari (fig. 19 bis).

L' impedenza apparente di ciascun avvolgimento del T. S., in quanto costituisce un ramo derivato fra due nodi a tensione costante, è :

$$
\mathbf{Z}_{0}{ }^{\prime}=r_{0}{ }^{\prime}-j \lambda_{0}{ }^{\prime}=r_{0}\left(1+\mu_{0}{ }^{2} y_{0}{ }^{2}\right)-j \lambda_{0}\left(1-\mu_{0}^{2} y_{0}{ }^{2}\right)=1 / \mathbf{Y}_{0}^{\prime},
$$

e quindi l'ammettenza apparente:

$$
\mathbf{Y}_{0}^{\prime}=\frac{\mathbf{Y}_{0}}{1+\mu_{0}^{2} \mathbf{Y}_{0}^{2}}=g_{0}^{\prime}+j b_{0}^{\prime} \text {. }
$$

$L$ 'impedenza originaria $Z_{0}$ può venir diminuita se si inserisea un condensatore opportuno nel ramo del T. $\mathbf{S}$.

L' impedenza apparente di ciascun avvolgimento del T. D, a rotore in moto, è, secomdo le (13), § 3,

ove :

$$
\mathbf{Z}_{1 \mathrm{k}}=r_{1 \mathrm{k}}-j \lambda_{1 \mathrm{k}}=1 / \mathbf{Y}_{1 \mathrm{k}}
$$

$$
\frac{\lambda_{1 \mathrm{k}}}{r_{\mathrm{lk}}}=\frac{\lambda_{1}{ }^{\prime}-\mathrm{K}^{2}\left[b_{1}{ }^{\prime}\left(g^{\prime 2}-b^{\prime 2}\right)+g_{1}{ }^{\prime} 2 b^{\prime} g^{\prime}\right]}{r_{1}^{\prime}+\mathrm{K}^{2}\left[g_{1}^{\prime}\left(g^{\prime 2}-b^{\prime 2}\right)-b_{1}^{\prime} 2 b^{\prime} g^{\prime}\right]}=\frac{\operatorname{sen} \alpha_{1}{ }^{\prime}-\mathrm{P}^{9} \operatorname{sen}\left(2 \alpha^{\prime}+\alpha_{1}{ }^{\prime}\right)}{\cos \alpha_{1}{ }^{\prime}+\mathrm{P}^{2} \cos \left(2 \alpha^{\prime}+\alpha_{1}{ }^{\prime}\right)}
$$

quiindi, l'ammettenza apparente (dinamica)

$$
\mathbf{Y}_{1 \mathrm{k}}=\frac{\mathbf{Y}_{1}^{\prime}}{1+\mathrm{K}^{2}} \overline{\mathbf{Y}^{\prime 2} \mathbf{Y}_{1}^{\prime 2}}
$$

Le espressioni simboliche $\mu_{0} \mathbf{Y}_{0}$ e $K \mathbf{Y}^{\prime} \mathbf{Y}_{1}^{\prime}$ dei rapporti di trasformazione nei due singoli apparecchi,

$$
\mu_{0} Y_{0}=P_{0}\left(\cos \alpha_{0}+j \operatorname{sen} \alpha_{0}\right) \quad, \quad K Y^{\prime} Y_{1}^{\prime}=\mathrm{P}(\cos a+j \operatorname{sen} a),
$$


hanno $\mathrm{i}$ moduli : $p_{0}=\mu_{0} y_{0}<1, \quad \mathrm{P}=\mathrm{K} y^{\prime} y^{\prime}$;

e gli argomenti :

$$
\alpha_{0}=\operatorname{arctg} \frac{b_{0}}{g_{0}},\left(\alpha_{1}^{\prime}+\alpha^{\prime}\right)=a=\operatorname{arctg} \frac{\left(b_{1}^{\prime} g^{\prime}+g_{1}^{\prime} b^{\prime}\right)}{\left(g^{\prime} g_{1}-b^{\prime} b_{1}^{\prime}\right)},
$$

i quali, aumentati di $\pi / 2$, rappresentano i ritardi delle f. e. $m$. indotte secondarie sulla $V$ primaria.

Si avranno le equazioni del sistema, scrivendo le espressioni simboliche delle cadute di tensione nefle impedenze dei quattro avvolgimenti, in funzione delle due tensioni terminali e dei rapporti di trasformazione singoli :

Nei primarî :

$$
\mathbf{J}_{0_{1}} \mathbf{Z}_{0}^{\prime}=\mathbf{V}-j \mu_{0} \mathbf{Y}_{0} \cdot \mathbf{V}_{e} ; \mathbf{J}_{1} \mathbf{Z}_{1 \mathrm{k}}=\mathbf{V}-j \mathbf{K} \mathbf{Y}^{\prime} \mathbf{Y}_{\mathfrak{1}}^{\prime} \cdot \mathbf{V}_{e}
$$

nei secondari :

$$
\mathbf{J}_{02} \mathbf{Z}_{0}^{\prime}=j \mu_{0} \mathbf{Y}_{0} \cdot \mathbf{V}-\mathbf{V}_{e} ; \boldsymbol{d}_{2} \mathbf{Z}_{1 \mathrm{k}}=j \mathbf{K} \mathbf{Y}^{\prime} \mathbf{Y}_{\mathbf{i}}^{\prime} \cdot \mathbf{V}-\mathbf{V}_{e}
$$

Sa ne ricavano: le quattro correnti interne; le correntí esterne di linea

$$
\mathbf{J}_{p}=\mathbf{J}_{01}+\mathbf{J}_{1}, \quad \mathbf{J}_{e}=\mathbf{J}_{02}+\mathbf{J}_{2}
$$

e la tensione secondaria :

$$
\mathbf{V}_{e}=\mathbf{J}_{e} \mathbf{Z}_{e}=\mathbf{V} \frac{j \mu_{0} \mathbf{Y}_{0} \cdot \mathbf{Y}_{0}^{\prime}+j \mathbf{K} \mathbf{Y}^{\prime} \mathbf{Y}_{1}^{\prime} \cdot \mathbf{Y}_{1 \mathrm{k}}}{\mathbf{Y}_{e}+\mathbf{Y}_{\mathbf{0}}^{\prime}+\mathbf{Y}_{\mathbf{1 k}}}
$$

Il rapporto di trasformazione delle tensioni a vuoto risultante $\left(\nabla_{e} / V\right)_{0}$ è il modulo della $(39)$, postavi aguale a zero l'ammettenza esterna $\mathbf{Y}_{e}=g_{e}+j b_{e}$, che influisce d'altra parte sul solo denominatore.

Il calcolo di esso si eseguisce sostituendo la (36) e le abbreviazioni seguenti :

$$
\begin{aligned}
& g_{0} g_{0}{ }^{\prime}-b_{0} b_{0}{ }^{\prime}=\mathrm{G}_{0}{ }^{2} \quad g^{\prime} g_{1}^{\prime}-b^{\prime} b_{1}^{\prime}=\mathrm{G}_{1}{ }^{2} \quad g_{0}{ }^{\prime} g_{1}{ }^{\prime}+b_{0}{ }^{\prime} b_{1}^{\prime}=\sigma^{2} \\
& g_{0} b_{0}{ }^{\prime}+g_{0}{ }^{\prime} b_{0}=\mathrm{B}_{0}{ }^{2} \quad g^{\prime} b_{1}{ }^{\prime}+g_{1}{ }^{\prime} b^{\prime}=\mathrm{B}_{1}{ }^{2} \quad g_{0}{ }^{\prime} b_{1}{ }^{\prime}-g_{1}{ }^{\prime} b_{0}{ }^{\prime}=+\delta^{2} \\
& \mathrm{G}_{0}^{4}+\mathrm{B}_{0}^{4}=y_{0}{ }^{2}{y_{0}}^{\prime 2} \quad \mathrm{G}_{1}{ }^{4}+\mathrm{B}_{1}{ }^{6}=y^{\prime 2} y_{1}{ }^{2} \quad \sigma^{4}+\delta^{4}=y_{0}{ }^{2} y_{1}{ }^{2} \\
& \operatorname{tg}\left(\alpha_{0}+\alpha_{0}{ }^{\prime}\right)=B_{0}{ }^{2} / G_{0}{ }^{2} \operatorname{tg}\left(\alpha_{1}{ }^{\prime}+\alpha^{\prime}\right)=B_{1}{ }^{2} / G_{1}{ }^{2} \operatorname{tg}\left(\alpha_{1}{ }^{\prime}-\alpha_{0}{ }^{\prime}\right)=\delta^{2} / \sigma^{2} .
\end{aligned}
$$


Si realizza così l'espressione :

$$
\left(\begin{array}{l}
V_{e} \\
-V
\end{array}\right)=\sqrt{\frac{\mathrm{P}^{4} P_{0}^{2} y_{0}^{\prime 2}+2 \mu_{0} \mathrm{~K}^{3} \cdot \alpha+\mathrm{P}^{2} \cdot P+2 \mu_{0} \mathrm{~K} \cdot \gamma+\mathrm{P}_{0}^{\prime} y_{0}^{\prime 2}}{\mathrm{P}^{4} y_{0}^{\prime 2}+2 \mathrm{P}^{2} \cdot \eta+\left(g_{0}^{\prime}+g_{1}^{\prime}\right)^{2}+\left(b_{0}^{\prime}+b_{1}^{\prime}\right)^{2}}},
$$

ove si è posto :

$$
\begin{aligned}
& \bar{\alpha}=y^{\prime 2} y_{1}{ }^{4}\left(g^{\prime} \mathrm{G}_{\mathrm{\theta}}{ }^{2}-b^{\prime} \mathrm{B}_{0}{ }^{2}\right)=y^{\prime 2} y_{1}{ }^{\prime k} y_{0} y_{0}{ }^{\prime} y^{\prime} \cos s ; \\
& \bar{\gamma}=\mathrm{G}_{1}{ }^{2}\left(g_{1}{ }^{\prime} \mathrm{G}_{0}{ }^{2}+b_{1}{ }^{\prime} \mathrm{B}_{0}{ }^{2}\right)-\mathrm{B}_{0}{ }^{2}\left(b_{1}{ }^{\prime} \mathrm{G}_{0}{ }^{2}-g_{1}{ }^{\prime} \mathrm{B}_{0}{ }^{2}\right)=y_{1}{ }^{\prime} y_{0} y_{0}{ }^{\prime} y^{\prime} \cos (2 a-s) \text {; }
\end{aligned}
$$

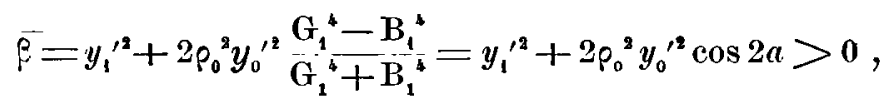

$$
\begin{aligned}
& \vec{\eta}=\frac{\mathrm{G}_{1}{ }^{4}-\mathrm{B}_{1}{ }^{4}}{\mathrm{G}_{1}{ }^{4}+\mathrm{B}_{1}{ }^{4}}\left(y_{0}{ }^{2}+\sigma^{2}\right)+\underset{\mathrm{G}_{1}+\mathrm{B}_{1}{ }^{4}{ }^{2} \mathrm{G}^{2}}{2{ }^{2}}=y_{0}{ }^{2} \cos 2 a+ \\
& +y_{0}^{\prime} y_{1}^{\prime} \cos (2 a-d)<0
\end{aligned}
$$

abbreviando con :

$$
a=\alpha_{1}^{\prime}+\alpha^{\prime}=\text { ritardo della corrente dinamicamente in- }
$$
dotta sul rotore, rispetto alla tensione primaria applicata; $a$ più prossimo a $3 \pi / 2$ che $\mathrm{a} \pi ; 2 a$ congrnente a $\pi$;

$$
\begin{aligned}
& s=\alpha_{0}+\alpha_{0}^{\prime}+\alpha^{\prime}==\left(\alpha_{0}+\pi / 2+\alpha_{0}{ }^{\prime}\right)-\left(\pi / 2-\alpha^{\prime}\right) ; \\
& 2 a-s=\left(2 \alpha_{1}^{\prime}+\alpha^{\prime}+\pi / 2\right)-\left(\alpha_{0}+\pi / 2+\alpha_{0}^{\prime}\right) ; \\
& d=\alpha_{1}^{\prime}-\alpha_{0}^{\prime}=\operatorname{arctg} \delta^{2} / \sigma^{2}=\operatorname{arctg} b_{1}^{\prime}\left|g_{1}^{\prime}-\operatorname{arctg} b_{0}^{\prime}\right| g_{0}^{\prime}>0 .
\end{aligned}
$$

Il rapporto di trasformazione (40) pertinente al sistema dei due trasformatori in parallelo, per $K$ (o $P$ ) crescente da zero, parte dal valore

$$
\mathrm{P}_{0}^{\prime}=\frac{\rho_{0} y_{0}^{\prime}}{\sqrt{y_{0}^{\prime 2}+y_{1}^{\prime 2}+2 \sigma^{2}}}<\rho_{0}
$$

con un gradiente avente il segno di $\mu_{0} K \cdot \vec{\gamma}$. Supposto $\mu_{0} K>0$, - ciò che si può sempre ottenere, per un collegamento qualunque dei due apparechi, scegliendo il verso della velocità, - il gradiente iniziale ha il segnó del coefficiente $\bar{\gamma}$. Allora, per $\bar{\gamma} \gtrless 0$, la funzione (40) è inizialmente crescente, oppure comincia col diminuire fino ad un minimo per poi risalire verso un massimo, e in seguito decresce assintoticamente verso $\rho_{0}$; (fig. 20 , curva punteggiata). 
Per lo scopo che perseguiamo, interessa fissare le condizioni che rendono questo rapporto di trasformazione crescente fin dall'origine. Affinchè $\gamma$ sia positivo, richiedesi che l'angolo $(2 a-s)$ cada nel primo o nel quarto quadrante:

$\left(40^{*}\right) \quad h \pi+\pi / 2>\left(2 \alpha_{1}{ }^{\prime}+\alpha^{\prime}+\pi / 2\right)-\left(\alpha_{0}+\alpha_{0}{ }^{\prime}+\pi / 2\right)>3 \pi / 2+2 h \pi$.

Ad interpretare questa condizione, giova trasformare la (40) sostituendovi i valori dei coefficienti in funziome degli angoli, il che permette di conglobare insieme $i$ due termini di grado dispari al numeratore, nonchè $i$ vari termini di grado pari, ottenendo dapprima: $\mathrm{P}_{\mathfrak{n}}=$

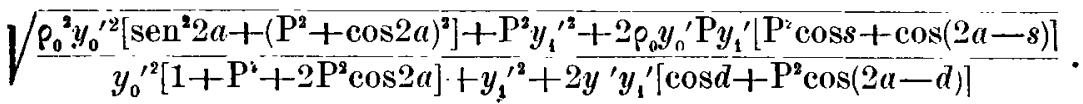

Dividendo allora sopra e sotto per il fattore d'impecienza, $\left(16^{*}\right)$,

$$
\sqrt{1+\mathrm{P}^{4}+2 \mathrm{P}^{2} \cos 2 a}=z_{1 \mathrm{k}} / z_{\mathrm{i}}^{\prime}=y_{\mathrm{i}}{ }^{\prime} \mid y_{1 \mathrm{k}}
$$

si giunge alla forma più generale di $\left(V_{e} / V\right)_{0}$ :

$$
\mathrm{P}_{v}=\sqrt{\frac{\rho_{0}^{2} y_{0}^{\prime 2}+\mathrm{P}^{2} y_{1 \mathrm{k}}{ }^{2}+2 \rho_{0} y_{0}{ }^{\prime} \mathrm{P} y_{1 \mathrm{k}} \cos \left(\operatorname{arctg} \frac{\operatorname{sen} 2 a}{\mathrm{P}^{2}+\cos 2 a}-s\right)}{y_{0}{ }^{2}+y_{1 \mathrm{k}}{ }^{2}+2 y_{0} y_{1 \mathrm{k}} \cos \left(d-\operatorname{arctg} \frac{\mathrm{P}^{2} \operatorname{sen} 2 a}{1+\mathrm{P}^{2} \cos 2 a}\right)}} .
$$

quozionte della somma vettoriale delle correnti indotte (per nn Volt efficace applicato ai primari) alla somma vettoriale delle ammettenze dei singoli secondari, (cfr. pag. 35).

L'angolo, di cui il cos è al numeratore, è la differenza di fase con la quale le due correnti indotte, $\mathrm{V} \rho_{0} y_{0}{ }^{\prime}$ e $\mathrm{VP} \mathbf{y}_{1 \mathrm{x}}$, si presentano ai nodi secondari: $\left(\varphi_{2}-\varphi_{02}\right)$. L'angolo corrispondente nel denominatore, divergenza angolare fra le ammettenze secondarie, è : $d-\operatorname{arctg} \ldots=\operatorname{arctg} \lambda_{1 \mathrm{k}} / r_{1 \mathrm{k}}-\alpha_{0}^{\prime}=\alpha_{1}^{\prime}-\operatorname{arctg} \frac{\mathrm{P}^{2} \operatorname{sen} 2 a}{1+\mathrm{P}^{\prime} \cos 2 a}-\alpha_{0}^{\prime}$.

La differenza fra questi due angoli, variabili con la velocità, è costante :

$$
\left(\varphi_{2}-\varphi_{02}\right)-\left(\alpha_{1 k}-\alpha_{0}{ }^{\prime}\right)=a-\alpha_{0} .
$$


L'angolo $\varphi_{02}$ è il ritardo, nel T. S., della corrente indotta secondaria (o di corto eircuito) sulla tensione primaria applicata :

$$
\begin{gathered}
\varphi_{03}=\alpha_{0}{ }^{\prime}+\pi / 2+\alpha_{0}=\alpha_{0}+\operatorname{arctg} \cdot \frac{1+\rho_{0}{ }^{2}}{1-\rho_{0}{ }^{2}} \operatorname{tg}\left(\alpha_{0}+\pi / 2\right)= \\
=\frac{\pi}{2}+\operatorname{arctg} \frac{\operatorname{sen} 2 \alpha_{0}}{\rho_{0}{ }^{2}+\cos 2 \alpha_{0}} .
\end{gathered}
$$

ove $\alpha_{0}+\pi / 2=\overline{\mathrm{E}_{02} \mathrm{~V}}=\varepsilon_{02}$ è il ritardo della f. e. m. indotta sulla tensione primaria.

L'angolo $\varphi_{s}$ è il ritardo, nel T. D., della corrente indotta secondaria (o di c. c.) sulla tensione primaria, (19):

$$
\varphi_{2}=\varphi_{1}+\left(\alpha_{1}^{\prime}+\alpha^{\prime}+\pi / 2\right)=\alpha_{1 \mathrm{k}}+\overline{\mathrm{E}_{2} \bar{\nabla}}=\alpha_{1 \mathrm{k}}+\varepsilon_{2} .
$$

Mentre $\varphi_{02}$ \& costante, $\varphi_{2}$ va decrescendo, col crescere della velocità di rotore, dal valore iniziale $\left(2 \alpha_{i}^{\prime}+\alpha^{\prime}+\pi / 2\right)=$ $=\left(a+\pi / 2+\alpha_{1}^{\prime}\right) ;$ come mostrano le espressioni :

$$
\digamma_{2}=\alpha_{1}^{\prime}+\operatorname{arctg} \cdot \frac{1+\mathrm{P}^{2}}{1-\mathrm{P}^{2}} \operatorname{tg}(a+\pi / 2), \text { ore } a+\pi / 2=\overline{\mathrm{E}_{2} \mathrm{~V}}=\varepsilon_{\mathrm{a}},
$$

$$
=2 x_{1}^{\prime}+\alpha^{\prime}+\pi / 2-\operatorname{arctg} \frac{\mathrm{P}^{2} \operatorname{sen} 2 a}{1+\mathrm{P}^{\prime} \cos ^{2} 2 a}=\frac{\pi}{2}+\operatorname{arctg} \frac{\operatorname{sen} 2 a}{\mathrm{P}^{2}+\cos 2 a}-\alpha^{\prime} \text {. }
$$

L'angolo $\varphi_{3}-p_{02}$, differenza fra i ritardi globali nei due trasformatori, vale dunque (41) la differenza di fase fra le f. e. m. indotte più la divergenza angolare delle ammettenze secondarie :

$$
\begin{aligned}
& \text { 41') } \quad \varphi_{3}-\varphi_{03}=\operatorname{arctg} \frac{\operatorname{sen} 2 a}{\mathrm{P}^{2}+\cos 2 a}-\alpha^{\prime}-\operatorname{arctg} \frac{\operatorname{sen} 2 \alpha_{0}}{\rho_{0}^{2}+\cos 2 \alpha_{0}}= \\
& =\left(\varepsilon_{2}+\alpha_{1 \mathrm{k}}\right)-\left(\varepsilon_{03}+\alpha_{0}{ }^{\prime}\right)=\left(\varepsilon_{2}-\varepsilon_{02}\right)+\left(\alpha_{1 \mathrm{k}}-\alpha_{0}{ }^{\prime}\right) ; \quad \varepsilon_{2}-\varepsilon_{02}=a-\alpha_{0} . \\
& \quad \text { Ora, }\left(\varphi_{2}-\varphi_{08}\right), \text { partendo dal valore iniziale }
\end{aligned}
$$

$$
(2 a-s)=\left(2 \alpha_{1}^{\prime}+\alpha^{\prime}+\pi / 2\right)-\left(\alpha_{0}+\alpha_{0}{ }^{\prime}+\pi / 2\right),
$$

va diminuendo col crescere della velocità, insieme all'angolo

il quale parte da

$$
\alpha_{1 \mathrm{r}}-\alpha_{0}^{\prime}=d-\operatorname{arctg} \cdot \mathrm{P}^{2} \operatorname{sen} 2 a /\left(1+\mathrm{P}^{2} \cos 2 a\right),
$$

$$
\alpha_{1}^{\prime}-\alpha_{0}^{\prime}=(2 a-s)-\left(\varepsilon_{2}-\varepsilon_{02}\right) ;
$$

e la loro differenza rimane costante, $\left(\varepsilon_{2}-\varepsilon_{02}\right)$. 
La somma vettoriale delle correnti indotte sarà crescente dall'origine, per $\rho_{0}$ e $P$ dello stesso segno e $\bar{\beta}>0$, se abbiasi $\cos (2 a-s)>0,\left(40^{*}\right)$; e tale si manterrà per qualunque valore di $\mathrm{P}^{2}$. D'altra parte, l' ammettenza complessiva dei secondari è funzione decrescente dall'origine, verso un minimo, se $\cos 2 a<0$. Quindi la funzione $\mathrm{P}_{v}$ sarà crescente dall'origine verso un massimo, offrendo nel primo ramo della sua curva una buona legge di amplificazione alla supposta grandezza sinoidale. Con 1'ugnaglianza $\bar{\gamma}=0,2 a-s=(2 h+1) \pi / 2$, il ritardo globale iniziale nel T. D. (che è invero dell'ordine di $\left.2 \pi+\alpha_{1}{ }^{\prime}\right)$ supera di $3 \pi / 2$ il ritardo globale costante che caratterizza il T. S. (dell'ordine di $\pi$ ), e la funzione parte da $P^{\prime}{ }_{0}$ con gradiente nullo.

Un caso interessante si ha infine quando sieno nulli entrambi i coefficienti $\alpha$ e $\gamma$ nella $(40)$; non rimangono allora che $\mathrm{i}$ termini a potenze pari della velocita e di $\rho_{0}$ : il funzionamento diviene indipendente dal modo delle connessioni $e$ dal verso della velocità.

Per questo non si richiede che di aggiungere alla condizione precedente anche la $\left(21^{*}\right), \mathrm{G}_{1}{ }^{2}=0$, inerente al solo T. D. In sostanza, bisognerà avere:

$\left(40^{*}\right)_{1,2} \frac{\mathrm{B}_{0}^{2}}{\mathrm{G}_{0}^{2}}=\frac{b_{\mathrm{i}}^{\prime}}{g_{\mathrm{i}}^{\prime}}=\frac{g^{\prime}}{b^{\prime}}$, ossia angolarmente $: a=s=\frac{\pi}{2}(2 h+1)$.

La prima di queste condizioni significa $\alpha_{1}^{\prime}=\alpha_{0}+\alpha_{0}^{\prime}$, ossia: $\left(10^{*}\right)_{1} \quad \rho_{0}^{2}=\frac{\operatorname{sen}\left(\alpha_{0}-\alpha_{1}^{\prime}\right)}{\operatorname{sen} \alpha_{1}^{\prime}}, \quad$ ossia $\quad \frac{2 \lambda_{0} r_{0}}{r_{0}^{2}-\left(\lambda_{0}^{2}-\mu_{0}^{2}\right)}=\frac{\lambda_{1}^{\prime}}{r_{1}^{\prime}}$, e potrà sempre effettuarsi se il 'T. S. sia a riluttanza variabile, oppure mediante inserzione di capacità. Notisi che

$$
\alpha_{0}+\alpha_{0}{ }^{\prime}<\pi / 2 \text { se } r_{0}{ }^{2}>\lambda_{0}{ }^{2}-\mu_{0}{ }^{2} \text {. }
$$

Detta condizione vuole : $g_{0} \delta_{0}{ }^{3}-b_{0} \sigma^{2}=0,\left(d=\alpha_{0}\right)$; e quindi esclude $\delta^{2}=0$.

Con la seconda conaizione, $\alpha_{1}^{\prime}+\alpha^{\prime}=3 \pi / 2$, la f. e. $\mathrm{m}$. indotta secondaria nel T. D. raggiunge la fase della tensione 
primaria $\overline{\mathrm{E}_{2} \mathrm{~V}}=\varepsilon_{2}=2 \pi$, e $\varphi_{2}$ acquista il valore costante $2 \pi+\alpha_{1}^{\prime}$. Ne risulta $\rho_{2}-\varphi_{02}=3 \pi / 2, \alpha_{1 \mathrm{k}}-\alpha_{0}^{\prime}=d=\alpha_{0}$. A conseguire le due condizioni, converrà che il T. D. sia molto reattivo $\left(2 \alpha_{0}>\alpha_{1}^{\prime}>\alpha_{0}\right)$.

Supponendo adunque che i due apparecchi soddisfino le $\left(40^{*}\right)_{1,2}$, il rapporto di trasformazione a vuoto si riduce alla forma :

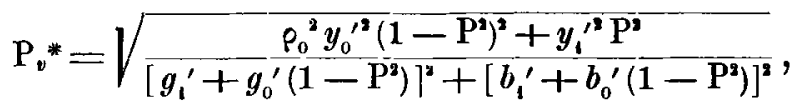

che conviene anche scrivere, a velocità avanzata,

$$
\mathrm{P}_{v}^{*}=\sqrt{\frac{P_{0}^{2} y_{0}^{\prime 2}\left(\mathrm{P}^{2}-1\right)^{2}+y_{1}^{\prime 2}\left(\mathrm{P}^{1}-1\right)+y_{1}{ }^{2}}{{y_{0}^{\prime 2}}^{2}\left(\mathrm{P}^{2}-1\right)^{2}+2 \sigma^{2}\left(\mathrm{P}^{2}-1\right)+y_{1}^{\prime 2}}}
$$

Questa forma, con $\mathrm{P}$ crescente, parte dal valore $P_{0}^{\prime}$ con gradiente nullo e assume tosto un andamento crescente. Passa per il valore 1 avviandosi rapidamente ad un massimo acuto, dopo di che ridiscende all'unita per tendere infine assintoticamente a $\rho_{0}$, (fig. 20, curva $\mathrm{P}_{v}^{*}$ ).

Le ascisse di $\mathrm{P}_{v}^{*}=1$ son date da

$$
\mathrm{P}^{2}=1+\frac{y_{1}^{\prime 2}+2 \sigma^{2}}{2 y_{0}^{\prime 2}\left(1-\rho_{0}^{2}\right)}(1 \mp 1)
$$

e cioè sono:

$$
\mathrm{K}^{\prime}=z^{\prime} z_{1}^{\prime} \quad, \quad \mathrm{K}^{\prime \prime}=z^{\prime} z^{\prime}{ }_{1} \sqrt{\frac{\left(g_{0}^{\prime}+g_{1}\right)^{2}+\left(b_{0}^{\prime}+b_{1}\right)^{2}-\rho_{0}{ }^{2} y_{0}^{\prime 2}}{y_{0}{ }^{\prime 2}\left(1-\rho_{0}{ }^{2}\right)}} .
$$

Intermediariamente a questi valori uno, la funzione $P_{v} *$ raggiunge un massimo. Uguagliando a zero la derivata della (44') presa rispetto a $P^{2}-1=X^{2}$, si ha l'equazione

$$
\mathrm{X}^{4} y_{0}{ }^{2}\left(y_{1}{ }^{2}+2 \rho_{0}{ }^{2} \sigma^{2}\right)+2 X^{3} y_{0}{ }^{2} y_{1}{ }^{\prime 2}\left(1-\rho_{0}{ }^{2}\right)-y_{1}{ }^{2}\left(y_{1}{ }^{12}+2 \sigma^{2}\right)=0 \text {, }
$$

la cui radice maggiore, sempre positiva, sostituita in $\left(44^{\prime}\right)$

dà per l'espressione del massimo: (45) $\mathrm{P}_{v}^{*}{ }_{\max }=$ :

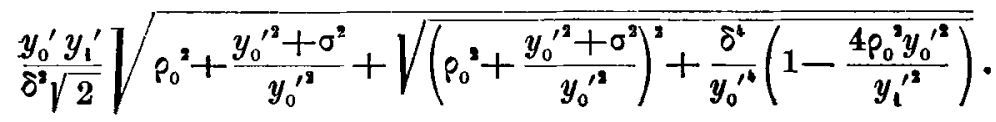


L'ascissa $K^{\prime \prime \prime}$ di questo massimo è più prossima a $K^{\prime}$ che a $K^{\prime \prime}$. L'esame della derivata seconda della (44') mostra che i due rami della curva, prima e dopo il massimo, sono estesamente convessi verso l'asse delle ascisse. La $\mathrm{P}^{*}{ }^{*}$ avrebbe quindi tutti i caratteri di una curva teorica di risonanza, salvo il massimo a $\infty$; questo è trattenuto a un valore finito dalla condizione stessa $\left(40^{*}\right)_{1}$, che esige sia

$$
\delta^{2}>0, b_{1}{ }^{\prime}\left|g_{1}{ }^{\prime}>b_{0}{ }^{\prime}\right| g_{0}{ }^{\prime},
$$

ossia la constante di tempo secondaria del $\mathrm{T}$. D. maggiore di quella del T. S.

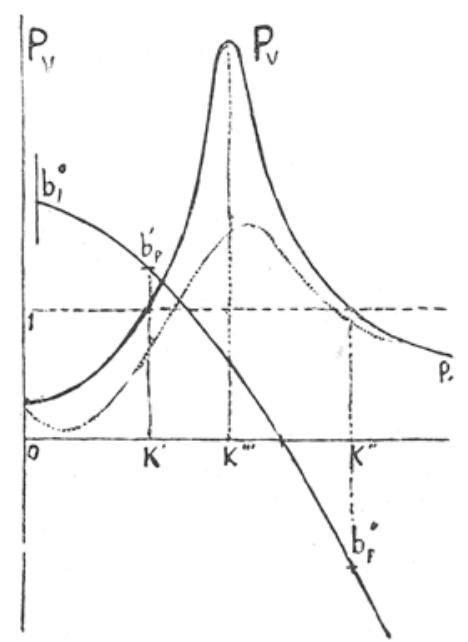

Fig. 20.

Che si tratti tuttavia di un caso analogo a quello della risonanza ottenibile con un condensatore, è dimostrato dal fatto che la elevazione della tensione secondaria $V_{e}$ è accompagnata dalla graduale scomparsa della corrente di magnetizzazione o componente reattiva della corrente totale $I_{p}$ che porta la tensione $\nabla$ ai nodi primari. Onde si deve ritenere che essa venga fornita dal $T$. $D$. 
Infatti : l'espressione simbolica di tale corrente, con $\mathbf{Y}_{e}=\mathbf{=}$,

$$
\mathbf{J}_{p}=\mathbf{V} \frac{\mathbf{Y}_{0} \mathbf{Y}_{0}^{\prime}+\mathbf{Y}_{1}^{\prime} \mathbf{Y}_{1 \mathrm{k}}+2 \mathbf{Y}_{0}^{\prime} \mathbf{Y}_{\mathrm{lk}}\left(1+\mu_{0} \mathbf{Y}_{0} \cdot \mathbf{K} \mathbf{Y}^{\prime} \mathbf{Y}_{1}^{\prime}\right)}{\mathbf{Y}_{0}^{\prime}+\mathbf{Y}_{1 \mathrm{k}}}
$$

posta sotto la forma canonica

$$
\mathbf{J}_{p}=\mathbf{V} \begin{aligned}
& \Gamma^{2}+j \mathbf{B}^{2} \\
& G+j B
\end{aligned}=\mathbf{V}\left(g_{p}+j b_{p}\right),
$$

fornisce la condizione affinchè essa si riduca alla sola componente attiva $\mathrm{V} g_{p}$ :

$$
b_{p}=\frac{B^{2} G-\Gamma^{2} B}{G^{2}+B^{2}}=0
$$

Con l'uso delle (36) e delle abbreviazioni solite, se ne ritrae una equazione del $4^{\circ}$ completa in $P$, la quale, con 1 ' introduzione delle condizioni $\left(40^{*}\right)_{1,3}$ si riduce alla forma:

$$
\begin{gathered}
\left(\mathrm{P}^{2}-1\right)^{2} b_{0} y_{0}{ }^{2}-\left(\mathrm{P}^{2}-1\right)\left(2 b_{1}^{\prime} y_{0}{ }^{2}+b_{1}{ }^{\prime} \sigma^{2}+g_{1}{ }^{\prime} \delta^{2}\right)+ \\
+2 \mu_{0} \mathrm{P}\left(\mathrm{P}^{2}-1\right)\left(\mathrm{B}_{0}{ }^{2} \delta^{3}-\mathrm{G}_{0}{ }^{3} \sigma^{2}\right)+2 \mu_{0} y_{1}{ }^{2} \mathrm{G}_{0}{ }^{2} \mathrm{P}+y_{1}{ }^{\prime 2}\left(b_{1}{ }^{\prime}+2 b_{0}{ }^{\prime}\right)=0
\end{gathered}
$$

Ora è sufficiente per noi il riconoscere che $b_{p}$ cambia di segno in un certo campo di variazione della velocità (o di P) o che questo campo è quello medesimo ove culmina l'elevazione della tensione secondaria.

L'espressione di $b_{p}$ è il rapporto fra il polinomio (46) e $\left(G^{2}+B^{2}\right)$ che è il denominatore stesso della (44). Sostituendovi i due valori di $\mathrm{P}\left(44_{1}\right)$ che corrispondono a $\mathrm{P}_{v}{ }^{*}=1$, si verifica che per la prima ascissa $\left(\mathrm{P}^{\prime}=1\right)$ si ha $b_{p}>0 ; e$ per la seconda ascissa : $b_{p}<0$. Vuol dire che nel campo intermedio esisterà un valore di $P$ reale e positivo che soddisfa la (46), ossia una certa velocità per la quale il « circuito $\gg$ dei due avvolgimenti primari è percorso dalla stessa corrente reattiva, sopperita dal T. D.

Per $\mathbf{P}^{\prime 2}=1$ si trova :

$$
\left(b_{p}\right)^{\prime}=b_{1}^{\prime}+2 b_{n}^{\prime}+2 \mu_{0} G_{0}^{3},
$$


grandezza positiva. La corrente $I_{p}$ ̀̀ ancora in ritardo di fase sulla tensione $V$. E per:

si trova :

$$
\mathbf{p}^{\prime \prime 2}=1+\frac{y_{1}^{\prime 2}+2 \sigma^{2}}{y_{0}^{\prime 2}\left(1-\rho_{0}^{2}\right)}=\frac{\left(g_{1}^{\prime}+g_{0}^{\prime}\right)^{2}+\left(b_{1}^{\prime}+b_{0}\right)^{2}-\rho_{0}^{2} y_{0}^{\prime 2}}{y_{0}^{\prime 2}\left(1-\rho_{0}^{2}\right)}
$$

$$
\left(b_{p}\right)^{\prime \prime}=\frac{b_{0}-\mathrm{A}\left[b_{1}{ }^{\prime}+2 b_{0}{ }^{\prime}+2 \mu_{0} \mathrm{G}_{0}{ }^{2} \mathrm{P}^{\prime \prime}\right]-\Delta\left[g_{1}{ }^{\prime}+2 g_{0}{ }^{\prime}-2 \mu_{0} \mathrm{~B}_{0}{ }^{2} \mathrm{P}^{\prime \prime}\right]}{[1-\Sigma]^{2}+\Delta^{2}}
$$

ove :

$$
\begin{aligned}
& \mathrm{A}=\frac{1-\rho_{0}^{2}}{\left(y_{1}^{\prime 2}+2 \sigma^{2}\right)^{2}}\left[\sigma^{4}\left(1+\rho_{0}^{2}+\frac{y_{1}^{\prime 2}}{\sigma^{2}}\right)-\delta^{4}\left(1-\rho_{0}{ }^{2}\right)\right]>0, \\
& \Delta=\frac{\delta^{2}\left(1-p_{0}{ }^{2}\right)}{y_{1}^{\prime 2}+2 \sigma^{2}}=\frac{\left(g^{\prime} b_{1}{ }^{\prime}-g_{1}{ }^{\prime} b_{0}{ }^{\prime}\right)\left(1-p_{0}{ }^{2}\right)}{g_{1}^{\prime}\left(g_{1}^{\prime}+2 g_{0}^{\prime}\right)+b_{1}^{\prime}\left(b_{1}^{\prime}+2 b_{0}^{\prime}\right)}>0, \\
& \Sigma=\frac{\sigma^{2}\left(1-P_{0}^{2}\right)}{y_{i}^{\prime 2}+2 \sigma^{2}} \cdot \quad\left(\Sigma^{2}+\Delta^{2}=\Sigma-A ; \quad A>\Delta\right) .
\end{aligned}
$$

La grandezza $\left(b_{p}\right)^{\prime \prime}$ ¿̀ dunque francamente negativa.

La compensazione della componente reattiva dei due primari è ora in eccesso e la corrente $I_{p}$ è in avanzo di fase sulla tensione $\mathrm{V}$. Fra $\mathrm{I}$ " e $\mathrm{P}^{\prime \prime}$ giace quiudi la radice reale positiva della (46) che interessa il nostro problema; essa non ha precisamente il valore che corrisponde al massimo della tensione secondaria, ma lo supera alquanto (fig. 20).

Rimane adunque dimostrato che, in determinate condizioni, un T. D. in parallelo con un T. S. è capace di elevare il suo fattore di potenza, innalzando la tensione secondaria e quindi la corrente di linea. Tali condizioni, $\left(40^{*}\right)_{1,2}$, riducono il T. D. ad un apparecchio prico di polaritì di fronte al T.S. che gli è associato e a fornire a quest'ultimo una corrente secondaria in quadratura; come farebbe un condensatore in parallelo.

\section{3. - Corrente di linea.}

Le due correnti secondarie (3s), in generale:

$$
\begin{gathered}
\mathbf{J}_{0 \mathbf{2}}=\mathbf{V} \mathbf{Y}_{0}{ }^{\prime j \mu_{0}} \mathbf{Y}_{0}\left(\mathbf{Y}_{e}+\mathbf{Y}_{\mathrm{lk}}\right)-j \mathrm{~K} \mathbf{Y}^{\prime} \mathbf{Y}_{1}^{\prime} \mathbf{Y}_{\mathrm{lk}} \\
\mathbf{Y}_{e}+\mathbf{Y}_{0}^{\prime}+\mathbf{Y}_{1 \mathrm{k}}
\end{gathered}
$$


si sommano sul circuito esterno nella corrente di linea $\mathbf{J}_{e}$, creando Ia teusione $V_{e}$ ai nodi secondari :

(38 $\mathbf{\Sigma}) \quad \mathbf{J}_{2}+\mathbf{J}_{v 3}=\mathbf{V}_{e} \mathbf{Y}_{e}=\mathrm{VY}_{e} \frac{j \mathbf{K} \mathbf{Y}^{\prime} \mathbf{Y}_{1}^{\prime} \mathbf{Y}_{1 \mathrm{k}}+j \mu_{0} \mathbf{Y}_{0} \mathbf{Y}_{0}^{\prime}}{\mathbf{Y}_{e}+\mathbf{Y}_{0}^{\prime}+\mathbf{Y}_{1 \mathrm{k}}}==\mathbf{J}_{e} ;$

e damuo luogo nel loro circuito alla corrente differenziale:

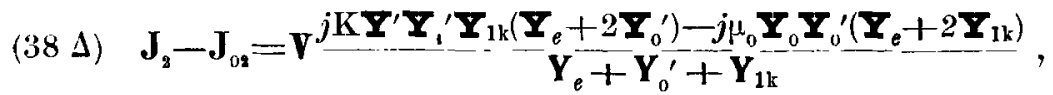
che più innanzi esamineremo.

La corrente secondaria di linea segue tutte le vicissitudini della tensione a vuoto $\left(\mathrm{V}_{e}\right)_{0}$ :

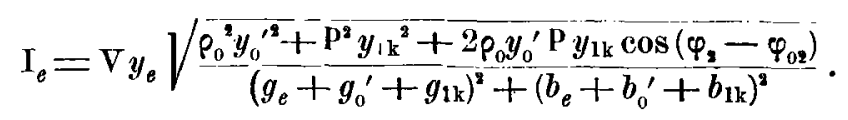

Per tutte quelle condizioni che rendono il doppio prodotto al numeratore positivo, la funzione è crescente fin dall' origine verso un massimo. Quando le due correnti indotte cadano in quadratura, $\left(40^{*}\right)_{1,2}$, il denominatore sotto radice diviene.

$$
\left(\left(g_{e}+g_{0}^{\prime}\right)\left(1-\mathbf{P}^{2}\right)+g_{1}^{\prime}\right)^{2}+\left(\left(b_{e}+b_{0}^{\prime}\right)\left(1-\mathrm{P}^{2}\right)+b_{1}^{\prime}\right)^{2}
$$

la curva di amplificazione della corrente di linea $I_{e}^{*}$ è analoga alla $P_{v}{ }^{*}$ della fig. 20 . Il suo massimo non soffre la limitazione imposta dalla $\left(40^{*}\right)_{1}$ che vuole $b_{1}{ }^{\prime} g_{0}{ }^{\prime}-b_{0}{ }^{\prime} g_{1}{ }^{\prime}>0$. Se si riduce infatti la $I_{e}^{*}$ a forma analoga con la $\left(44^{\prime}\right)$ e si derivi rispetto a $p^{2}-1=X^{2}$, si ha l'equazione per il massimo :

$$
\begin{aligned}
& \mathrm{X}^{4} y_{0}{ }^{\prime 2}\left(y_{1}{ }^{\prime 2} \frac{y_{e 0}{ }^{2}}{y_{0}{ }^{\prime 2}}+2 \rho_{0}{ }^{2} \sigma_{e 0}{ }^{2}\right)+2 \mathrm{X}^{2} y_{0}^{\prime 2} y_{1}{ }^{\prime 2}\left(\frac{y_{e c}{ }^{2}}{y_{0}{ }^{2}}-p_{0}{ }^{2}\right)-y_{1}^{\prime 2}\left(y_{1}^{\prime 2}+2 \sigma_{e 0}{ }^{2}\right)=0 \text {, } \\
& \text { ove: } y_{e 0}{ }^{2}=\left(g_{e}+g_{0}{ }^{\prime}\right)^{2}+\left(b_{e}+b_{0}{ }^{\prime}\right)^{2}, \sigma_{e 0}{ }^{2}=g_{1}{ }^{\prime}\left(g_{e}+g_{0}{ }^{\prime}\right)+b_{1}{ }^{\prime}\left(b_{e}+b_{0}{ }^{\prime}\right) \text {, } \\
& y_{1}{ }^{\prime 2} y_{e 0}{ }^{2}=\sigma_{e 0}{ }^{4}+\delta_{e 0}{ }^{4} \quad, \delta_{e 0}{ }^{2}=-b_{1}{ }^{\prime}\left(g_{e}+g_{0}{ }^{\prime}\right)-g_{1}{ }^{\prime}\left(b_{e}+b_{0}{ }^{\prime}\right) \text {. }
\end{aligned}
$$

La sua radice positiva sostituita in $\mathrm{I}_{e}^{*}$ da per l' espressione del massimo: (cfr. 45), (47*), $\mathrm{I}_{e^{*} \text { max }}=$

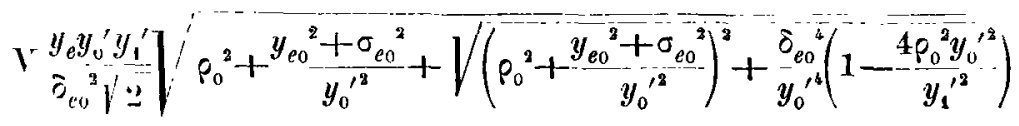


Se l'ammettenza di linea $g_{e}+j b_{e}$ sia tale da rendere evanescente $\delta_{e 0}{ }^{2}$, comunque sia grande $\delta^{2}=b_{1}{ }^{\prime} g_{0}{ }^{\prime}-g_{1}{ }^{\prime}{ }_{0}{ }^{\prime}$, questo massimo potra essere molto elevato $\left(\infty\right.$, per $\mathrm{p}^{2}-1=\sigma_{e 0}{ }^{2} / y_{e 0}{ }^{2}$ se $\delta_{e 0}{ }^{3}=0$ ossiase $\left.b_{1}{ }^{\prime} / g_{1}{ }^{\prime}=\left(b_{e}+b_{0}{ }^{\prime}\right)\left(g_{e}+g_{0}{ }^{\prime}\right)\right)$.

A creare la possibilità di casi consimili (rapporti di ammettenze o di impedenze che per una certa velocità divengono $\infty)$ basta però la condizione $\left(21^{*}\right)$ nel T. D.,

$$
a=\alpha_{1}^{\prime}+\alpha^{\prime}=3 \pi / 2,
$$

che rirluce il fattore $d$ 'impedenza

$$
z_{1 \mathrm{k}} / z_{\mathrm{i}}^{\prime}=\sqrt{1+\mathrm{P}^{4}+\overline{2}^{2}} \overline{\cos 2 a}^{\prime} \text { ad }\left(1-\mathrm{P}^{2}\right) \text { o a }\left(\mathrm{P}^{2}-1\right) \text {. }
$$

Anche se tale condizione limite non sia esattamente raggiunta, l'angolo $a=\left(\alpha_{i}^{\prime}+\alpha^{\prime}\right)$ è però sempre più prossimo a $3 \pi / 2$ che a $\pi$, onde $\cos 2 a, \cos \left(2 \alpha-\alpha_{1}{ }^{\prime}\right), \ldots$ sono $<0$, e il fattore d'impedenza $z_{1 \mathrm{k}} / z_{\mathfrak{1}}^{\prime}$ possiede un minimo, sen $2 a>0$, per $\mathrm{p}^{2}=-\cos 2 a$, che tende a zero per il caso limite.

La differenza di fase fra tensione secondaria $e$ tensione primaria, argomento del rapporto $\mathbf{V}_{e} / \mathbf{V},(39)$, è in generale:

$$
\begin{aligned}
& \dot{V_{e}} \bar{V}=\varphi_{03}=\operatorname{arctg} \frac{P y_{\mathrm{k}} \operatorname{sen}\left(a+\alpha_{\mathrm{k}}-\alpha_{0}-\alpha_{0}{ }^{\prime}\right)}{P_{0} y_{0}{ }^{\prime}+P y_{\mathrm{k}} \cos \left(\alpha+\alpha_{\mathrm{k}}-\alpha_{0}-\alpha_{0}{ }^{\prime}\right)}+ \\
& -\operatorname{arctg} \frac{b_{0}^{\prime}+b_{\mathrm{k}}+b_{e}}{g_{0}^{\prime}+g_{\mathrm{k}}+g_{e}}=\frac{\pi}{2}+\alpha_{0}+\operatorname{arctg} \frac{\mathrm{P} y_{\mathrm{k}} \operatorname{sen}\left(\varphi_{2}-\varphi_{02}\right)}{\rho_{0} y_{0}^{\prime}+\mathrm{P} y_{\mathrm{k}} \cos \left(\varphi_{2}-\varphi_{02}\right)}+ \\
& -\operatorname{arctg} \frac{y_{0}^{\prime} y_{\mathrm{k}} \operatorname{sen}\left(\alpha_{\mathrm{k}}-\alpha_{0}{ }^{\prime}\right)-g_{e} b_{0}^{\prime}+b_{e} g_{0}^{\prime}}{y_{0}^{\prime 2}+y_{0}^{\prime}} \frac{\alpha_{\mathrm{k}} \cos \left(\alpha_{\mathrm{k}}-\alpha_{0}{ }^{\prime}\right)+g_{e} g_{0}^{\prime}+b_{e} b_{0}^{\prime}}{} \text {. }
\end{aligned}
$$

Con $P$ crescente da zero, essa parte dal ritardo

$$
\varphi_{02}-\operatorname{arctg}\left(b_{0}{ }^{\prime}+b_{1}{ }^{\prime}+b_{e}\right) /\left(g_{0}{ }^{\prime}+g_{1}{ }^{\prime}+g_{e}\right)
$$

per diminuire verso un minimo, che può anche essere negativo (avanzo), risalendo infine assintoticamente verso il ritardo limite $\varphi_{02}-\operatorname{arctg}\left(b_{0}{ }^{\prime}+b_{e}\right) /\left(g_{0}^{\prime}+g_{e}\right)$ che, a vnoto, sarebbe quello $\left(\pi / 2+\alpha_{0}\right)$ spettante al T. S.

La condizione limite (21*) porta: $\overline{\mathrm{E}_{2} \overline{\mathrm{V}}}=2 \pi, \varphi_{2}=2 \pi+\alpha_{1}^{\prime}$. La presenza del $\mathbf{T}$. $S$. mantiene però la fase della tensione secondaria variabile con la velocità. 
Difatti (a vuoto, per semplificare) con detta condizione

$$
\left.y_{\mathrm{k}}=y_{\mathrm{k}}^{\prime} /\left(1-\mathrm{P}^{2}\right), \varphi_{2}-\varphi_{02}=3 \pi / 2+d-\alpha_{0}\right),
$$

la (48) assume la forma :

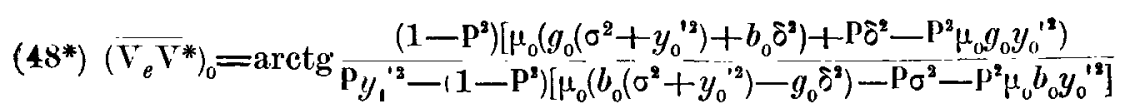

Variando fra gli stessi valori estremi come la (48), questa differenza di fase fra le due tensioni diviene un aranzo di fase nell' intervallo compreso fra le radici positive dei due fattori costituenti il numeratore. Tale intervallo comiucia da $P=1$, come $l^{\prime}$ intervallo $P^{\prime \prime}-P^{\prime},\left(44_{1}\right)$, entro il quale $P_{t}^{*}>1$ e la componente reattiva della corrente primaria, $V b_{p}$, cambia di segno, (46).

Se si aggiunge anche la condizione $\left(40^{*}\right)_{1},\left(b_{0} \sigma^{2}-g_{0} \delta^{2}=0\right.$, che porta $\left.b_{0} \delta^{2}+g_{0} \sigma^{2}=y_{0} y_{0}^{\prime} y_{1}^{\prime}\right)$, i due intervalli vengono a combaciare fra loro quasi completamente anche all'altra estremità.

In conclusione : il sistema dei due apparechi in parallelo, nelle dette condizioni particolari, possiede un intervallo di funzionamento nel quale la corrente totale primaria $I_{p}$ passa da ritardo ad avanzo di fase su $V$ e la tensione secondaria ¿ in avanzo sulla tensione primaria, pronta a dare in una ammettenza esterna una corrente $I_{e}$ possedente una amplifcazione ed un avanzo di fase corrispondenti.

\section{4. - Corrente differenziale.}

Non occorre insistere snlla necessità di certe relazioni costruttive condizionali, fra i due apparecehi S. e D. da farsi. funzionare in parallelo, sia tra grandezze assolute, sia di rapporti, se si vuole che gli effetti si sommino con una legge semplice. In pratica, non è permesso il funzionamento in parallelo di due (o più) trasformatori statici, se non abbiano uro stesso rapporto di trasformazione relle tensioni; ed è anche desiderabile che abbiano impedenze dello stesso ordine, od almeno uguali rapporti della reattanza alla resistenza 
(uguali costanti di tempo) negli avvolgimenti corrispondenti. Altrimenti, gli effetti si sommano in modo complicato e danneggevole, rendendo il funzionamento in realtà impraticabile.

Nel caso generale, come accade in tutti gli accoppiamenti in parallelo, circola fra $i$ due secondari una corrente differenziale. Anche a carico esterno nullo, questa corrente locale è tanto più intensa quanto pì̀ differenti fru loro sieno le costanti di tempo dei due secondari e non si annulla se non sia zero la forza elettromotrice indotta differenziale; ciò richiede che sieno uguali i rapporti di trasformazione nei due singoli apparecchi ed ugnali le costanti di tempo primarie (ovviamente, supposto di aver collegato in parallelo i secondari con polarità istantanee concordanti). Col carico secondario, specie se induttivo, la corrente differenziale generalmente aumenta. Questa corrente differenziale, la quale rappresenta potenza sottratta all'utilizzazione e localmente dissipata, praticamente non si annulla se non quando i due trasformatori sieno affatto identici.

Nel nostro caso, uno dei rapporti di trasformazione, $P_{0}, \grave{e}$ costante, l'altro, $\mathbf{P}$, cresce continnamente con la velocitì del rotore.

T' ha luogo a domandarsi se vi sia una velocitì che annulli la corrente differenziale. Anzitutto, a vuoto, tale corrente

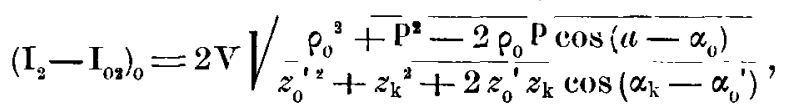

non svanisce se non sia nulla la f. e. m. differenziale, che è al numeratore; ciò che richiederebbe: $P= \pm P_{0}$ e rispettivamente $\cos \left(a-\alpha_{0}\right)= \pm 1$.

Con il carico esterno $\mathbf{X}_{e}=g_{e}+j b_{e}$, la corrente differenziale è il quoziente di una differenza vettoriale di cadute di tensione per la impedenza complessiva; ciò che equivale all'espressione della differenza vettoriale delle due correnti secondarie :

$$
2 \mathrm{~V} \sqrt{\rho_{0}^{2} y_{0}{ }^{2}}\left(\frac{y_{\mathrm{k} e}}{y_{0 \mathrm{k} e}}\right)^{2}+\mathrm{P}^{2} y_{\mathrm{k}}^{2}\left(\begin{array}{l}
y_{0 e} \\
y_{0 \mathrm{k} e}
\end{array}\right)^{2}-2 \rho_{0} y_{0} \mathrm{p}_{y_{\mathrm{k}}} \frac{y_{\mathrm{ke}} y_{0 e}^{\prime}}{y_{0 \mathrm{k} e}{ }^{2}} \cos \Delta \varphi
$$


ove : $\quad \Delta \varphi=\left(\varphi_{z}-\varphi_{02}\right)-\left(\varphi_{k_{e}}-\varphi_{0 e}\right)$;

il denominatore comune nei quozienti quadrati non è altro she l'ammettenza secondaria complessiva:

$$
\begin{aligned}
& y_{0 \mathrm{ke}}{ }^{2}=y_{\mathrm{u} e}{ }^{2}+y_{\mathrm{k} e}{ }^{2}+2 y_{\mathrm{e} e}{ }^{\prime} y_{\mathrm{k} e} \cos \left(\varphi_{\mathrm{k} e}-\varphi_{02}\right)= \\
& =\left(g_{0}^{\prime}+b_{\mathrm{k}}+g_{e}\right)^{2}+\left(b_{0}^{\prime}+b_{\mathrm{k}}+b_{e}\right)^{2},
\end{aligned}
$$

e le ammettenze parziali $y_{0 e}{ }^{\prime}$ ed $y_{k e}$ son definite da :

$$
\begin{aligned}
& y_{0 e}{ }^{\prime 2}=\left(\frac{g_{e}}{2}+g_{0}^{\prime}\right)^{2}+\left(\begin{array}{l}
b_{e} \\
2
\end{array}+b_{0}^{\prime}\right)^{2}, \varphi_{0 e}=\operatorname{arctg}\left(b_{e}+2 b_{0}^{\prime}\right) j\left(g_{e}+2 g_{0}^{\prime}\right) \\
& y_{\mathrm{k} e}{ }^{2}=\left(\frac{g_{e}}{2}+g_{\mathrm{k}}\right)^{2}+\left(\frac{b_{e}}{2}+b_{\mathrm{k}}\right)^{2}, \mathrm{f} \mathrm{k} e=\operatorname{arctg}\left(b_{o}+2 b_{\mathrm{k}}\right) /\left(g_{e}+2 g_{\mathrm{k}}\right) \text {. }
\end{aligned}
$$

Le due correnti secondarie sono tali come se ciascuna delle correnti indotte $V_{p_{0}} y_{0}^{\prime}$ e $V P_{y_{k}}$ si biforcasse in due circuiti derivati, rispettivamente di ammettenze

$$
\left(\mathbf{Y}_{e} / 2+\mathbf{Y}_{\mathrm{k}}\right) \text { e }\left(\mathbf{Y}_{e} / 2+\mathbf{Y}_{0}^{\prime}\right) ; \quad \text { cfr. }(38 \Delta) \text {. }
$$

Affinchè la corrente differenziale si annulli, ̀̀ necessario che le due correnti indotte VP $y_{k}$ e $V p_{0} y_{0}^{\prime}$ stieno fra loro come le ammettenze parziali $y_{k e}$ e $y_{0 e}$, e che la loro differenza di fase $\left(\varphi_{2}-\varphi_{02}\right)$ nguagli la divergenza angolare delle ammettenze stesse $\left(\varphi_{\mathrm{k} e}-\varphi_{0 e}\right)$ :

$$
\frac{P y_{\mathrm{k}}}{y_{\mathrm{k} e}}=\frac{P_{0} y_{0}^{\prime}}{y_{0 e}{ }^{\prime}} \quad, \quad \operatorname{tg}^{\prime}\left(\varphi_{2}-\varphi_{\mathrm{k} e}\right)=\operatorname{tg}\left(\varphi_{02}-\varphi_{0 e}\right) \text {. }
$$

Forme esplicite di queste equazioni di condizione si ottengono sostituendo espressioni gemelle per le grandezze omologhe nei due apparecchi, come le (42), (43) e le seguenti:

$$
\begin{aligned}
& g_{\mathrm{k}}=g_{1}^{\prime}\left(1+\mathrm{P}^{2} \frac{\cos \left(2 a-\alpha_{1}\right)}{\cos \alpha_{1}^{\prime}}\right) y_{\mathrm{k}}{ }^{2} / y_{1}^{\prime 2} \quad g_{0}^{\prime}=g_{0}\left(1+p_{0}^{2}\right) y_{0}^{\prime 2} / y_{0}^{2}
\end{aligned}
$$

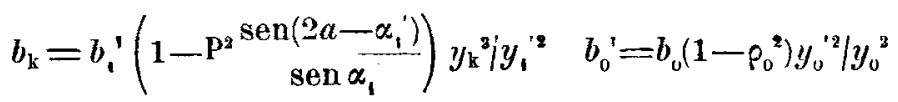

$$
\begin{aligned}
& y_{\mathrm{k}}{ }^{2}=y_{1}{ }^{2} /\left(1+\mathrm{P}^{4}+2 \mathrm{P}^{2} \cos 2 a\right) \quad y_{0}{ }^{3}=y_{0}{ }^{2} /\left(1-+\rho_{0}{ }^{4}+2 \rho_{0}{ }^{2} \cos 2 a_{0}\right) \text {. }
\end{aligned}
$$


Si giunge così, termine a termine, alle forme :

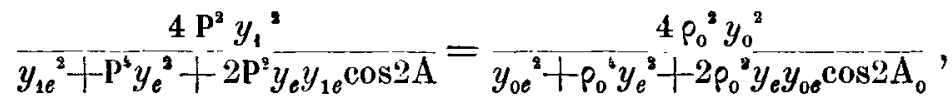

(50") $\operatorname{arctg} \frac{y_{1 e} \operatorname{sen} 2 \mathrm{~A}}{\mathrm{P}^{2} y_{e}+y_{1 e} \cos 2 \mathrm{~A}}-\alpha^{\prime}=\operatorname{arctg} \frac{y_{0 e} \operatorname{sen} 2 \mathrm{~A}_{0}}{\rho_{0}^{2} y_{e}+y_{0 e} \cos 2 \mathrm{~A}_{0}^{-}}, \operatorname{cfi} \cdot\left(41^{\prime}\right)$ ove :

$$
\begin{gathered}
y_{1 e}{ }^{2}=\left(g_{e}+2 g_{i}\right)^{2}+\left(b_{e}+2 b_{1}^{\prime}\right)^{2}, y_{0 e}{ }^{2}=\left(g_{e}+2 g_{0}\right)^{2}+\left(b_{e}+2 b_{0}\right)^{2} \\
2 A=2 a-\operatorname{arctg} \begin{array}{c}
2 y_{1} \operatorname{sen}\left(\alpha_{1}^{\prime}-\alpha_{e}\right) \\
y_{e}+2 y_{1} \cos \left(\alpha_{1}^{\prime}-\alpha_{c}\right)
\end{array}=2 a-\alpha_{1 e} \\
2 A_{0}=2 \alpha_{0}-\operatorname{arctg} \begin{array}{c}
2 y_{0} \operatorname{sen}\left(\alpha_{0}-\alpha_{e}\right) \\
y_{e}+2 y_{0} \cos \left(\alpha_{0}-\alpha_{e}\right)
\end{array}=2 \alpha_{0}-\alpha_{0 e} .
\end{gathered}
$$

La (50) esprime dunque l'uguaglianza delle cadute di tensione delle due correnti indotte sulle rispettive ammettenze (per 1 Volt efficace applicato al primario) e la $\left(50^{\prime}\right)$ '' opposizione delle loro fasi nel circuito comune.

Se l'angolo $\alpha$ sparisce, si ha il caso di due trasformatori statici $\left(2 \mathrm{~A}=2 \alpha_{1}^{\prime}-\alpha_{1 e}\right)$. Nel T. D. abbiamo però : $\pi<\alpha<3 \pi_{l}^{\prime} 2$.

Affinchè le (50) ammettano radici comuni, si richiederà in generale di soddisfare una equazione risultante fra i coefcienti delle due equazioni.

Da un lato, la (50) fornisce per il quadrato della velocità due valori :

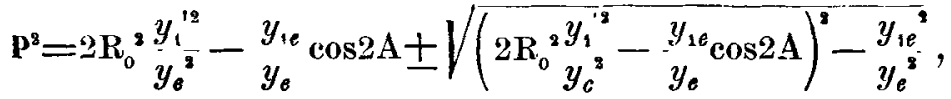

$$
\begin{aligned}
& \text { ossia }=\frac{y_{1 e}}{y_{e}}\left[\sqrt{\mathrm{R}_{0}^{2} y_{1}^{1_{1}^{2}}+\operatorname{sen}^{2} \mathrm{~A}} \pm \sqrt{\mathrm{R}_{0}^{2} y_{1 e}^{y_{1}^{\prime 2}}-\cos ^{2} \mathrm{~A}}\right]^{2},
\end{aligned}
$$

ove $R_{0}^{2}=y_{o e}^{\prime} / \rho_{0}^{2} y_{0}^{\prime 2}$ designa il reciproco del secondo membro della $(50)$.

Questi due valori sono reali positivi e diversi finchè si abbia $R_{0}^{2} y_{1}^{12}>y_{e} y_{10} \cos ^{2} \mathrm{~A}$, ovverosia

$$
\left(\frac{y_{0 e}-\rho_{0}^{2} y_{e}}{2 p_{0} y_{0}}\right)^{2}>\frac{y_{e} y_{1 e} \cos ^{2} \mathrm{~A}}{y_{1}^{\prime 2}}-\frac{y_{e} y_{0 e} \cos ^{2} \mathrm{~A}_{0}}{y_{0}^{2}},
$$


condizione tanto più largamente soddisfatta quanto più piccola sia l'ammettenza esterna $y_{e^{*}}$.

D'altro lato, la $\left(50^{\prime \prime}\right)$ dà per il quadrato della velocità il valore unico:

$$
\begin{aligned}
& \mathbf{P}^{2}=\frac{y_{1 e}}{y_{e}} \frac{y_{0 e} \operatorname{sen}\left(2 \mathrm{~A}-2 \mathrm{~A}_{0}\right.}{\left.\alpha^{\prime}\right)+p_{0}^{2} y_{e} \operatorname{sen}\left(2 \mathrm{~A}-\alpha^{\prime}\right)} \\
& \text { ossia : }==\begin{array}{l}
y_{1 e} \\
y_{e}
\end{array}\left[1+2 \cos ^{2} A\left(\operatorname{tg} A \operatorname{cotg}\left(A_{p}+\alpha^{\prime}\right)-1\right)\right] \text {, }
\end{aligned}
$$

ove $A_{\rho}$ sta per l'arctg secondo membro della (50"); $\left(A_{\rho}>A_{0}\right)$.

Per questa velocita, la corrente differenziale assume la forma $I^{2}-I_{0 z}$. Per le altre due velocità corrispondenti ai valori (51), la corrente stessa ha la forma $2 I_{y} \operatorname{sen} \varphi / 2$. L'intervallo fra questi valori e fra le correnti corrispondenti ̀̀ tanto minore quanto più piccola sia la disuguaglianza (51*) e si annulla insieme ad essa. Le due radici (51) si uguagliano allora nel valore

$$
\mathrm{p}^{3}=\frac{y_{1 e}}{y_{e}}=\sqrt{1+\frac{4 y_{1}^{\prime 2}}{y_{e}^{i}}+\frac{4 y_{i}^{i}}{y_{e}} \cos \left(\alpha_{i}-\alpha_{e}\right)} .
$$

Affinchè anche la (52) si riduca allo stesso valore, richiedesi :

$$
A=A_{\rho}+\alpha^{\prime}, \text { ossia } \operatorname{tg} A_{\rho}=\operatorname{tg}\left(\alpha_{1}^{\prime}-\alpha_{1 e} / 2\right) .
$$

Se ne deduce l'espressione dj

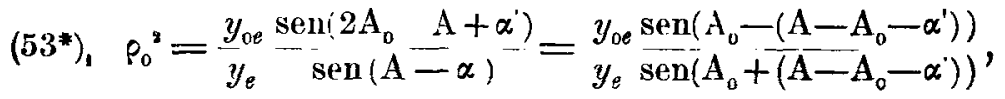

per eliminare $\rho_{0}$ dall'altra equazione di condizione

$$
\mathbf{R}_{0}^{2} y_{1}^{12}=y_{e} y_{1 e} \cos ^{2} \mathrm{~A} \text {, }
$$

ottenendone:

$$
\frac{y_{1}{ }^{\prime 2}}{y_{e} y_{1 e} \cos ^{2} \bar{A}}=\frac{y_{0}{ }^{2}}{y_{e} y_{0 e} \cos ^{2} \mathrm{~A}_{\mathrm{o}}}\left[1-\frac{\operatorname{sen}^{2}\left(\mathrm{~A}-\mathrm{A}_{0}-\alpha^{\prime}\right)}{\operatorname{sen}^{2} \mathrm{~A}_{0}}\right],
$$

relazione condizionale che lega insieme le quattro coppie di costanti originarie $b_{0} g_{0}, b_{1} g_{1}, b g, b_{e} g_{e}$, e che rappresenta, come le (50), l'uguaglianza in grandezze e fa-i delle cadute di tensione delle correnti nei secondari (per 1 Volt al pri- 
mario), quando la corrente differenziale debba annullarsi per il valore particolare $\mathrm{p}^{2}=y_{1 e} / y_{t}$.

Ora, scritte le (50) nella forma $\mathrm{P}^{4}-2 \mathrm{P}^{2} \chi_{0}+\chi^{2}=0$, $\mathbf{p}^{2}-\chi \xi=0$, ove : $\chi_{0}=2 \mathrm{R}_{0}{ }^{2} y_{1}{ }^{2} / y_{e}{ }^{2}-\chi \cos 2 \mathrm{~A} ; \chi=y_{1 e} / y_{e}$; e $\chi \xi$ è il secondo membro della (52); l'equazione risultante fra i coefficienti è : $\xi^{2}-2 \xi \chi_{0} / \chi+1=0$. Similmente eliminando $p_{0}{ }^{3}$ invece di $p^{2}$, si ha un'altra risultante della stessa forma. Queste due equazioni sono:

$$
=\left(\frac{y_{0 e}-\rho_{0}^{3} y_{e}}{2 \rho_{0} y_{0}}\right)^{2}-\left(y_{o e} \operatorname{sen} 2 A_{0}-\overline{A+\alpha^{\prime}-\rho_{0}^{2} y_{e} \operatorname{sen}} \frac{1-\alpha^{\prime}}{2 \rho_{0} y_{0} \operatorname{sen} A}\right)^{2}
$$

$\frac{y_{e} y_{1 e} \cos ^{2} \mathrm{~A}}{y_{1}{ }^{2}}-\frac{y_{e} y_{0 c} \cos ^{2} \mathrm{~A}_{0}}{y_{0}{ }^{2}}=$

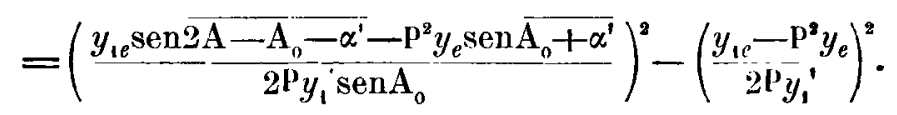

Entrambe tali risultanti si riducono alla (54) rispettivamente per le condizioni $\left(53^{*}\right)_{1}$ e (53), le quali annullano gli ultimi termini a destra. I prini termin: a sinistra dei secondí membri non possono invece annullarsi : avendo assunto $p_{0}<1$, si avrà sempre $y_{0 e}>p_{0}^{2} y_{e}$; perciò e conforme alla $\left(50^{\prime \prime}\right)$, sarì anche

$$
\frac{y_{1 e} \operatorname{sen} 2 \mathrm{~A}}{\overline{\mathbf{p}}^{2} y_{e}+y_{1 e} \cos 2 \mathrm{~A}}>\operatorname{tg}\left(\mathrm{A}_{0}+\alpha^{\prime}\right)
$$

e quindi per $\mathrm{p}^{2} y_{e} / y_{1 e}=1$, si richiede $\mathrm{A}>\mathrm{A}_{0}+\alpha^{\prime}$ ossia $A-\alpha>A_{0}$; che collima con quanto è prescritto dalle (53*), e (54) più restrittivamente $A_{0}>A-A_{0}-\alpha^{\prime}>0$ ovverosia: $\alpha_{0}-\alpha_{0 e} / 2>\left(\alpha_{1}^{\prime}-\alpha_{1 e} / 2\right)-\left(\alpha_{0}-\alpha_{0 e} / 2\right)>0$. Ad assolvere questa condizione ovvia, concorrono soltanto i sei elementi delle ammettenze $g_{0}+j b_{0}, g_{1}+j b_{1}, g_{e}+j b_{e}$ degli avvolgimenti e della linea. 
A conferma: ordinata la (56) secondo $\mathrm{P}^{2} y_{e}$ e risolta come biquadratica, si ricava l'espressione

$$
\begin{aligned}
& \mathbf{p}^{2}=\frac{y_{e} e}{y_{e}} \frac{\operatorname{sen}\left(2 A-2 A_{0}-\alpha^{\prime}\right)}{\operatorname{sen}\left(2 A_{0}+\alpha^{\prime}\right)} \chi^{\prime}
\end{aligned}
$$

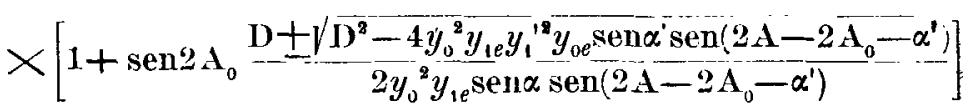

per il quadrato della velocità che in generale annulla la cor'rente differenziale, in funzione delle quattro coppie di costanti originarie. Si è abbreviato con

$$
\text { I) }=-y_{0}^{2} y_{1 e} \operatorname{sen} 2 \Lambda-y_{1}{ }^{2} y_{0 e} \text { ven } 2 A_{0} \text {. }
$$

La condizione di realità può tradursi nella forma seguente:

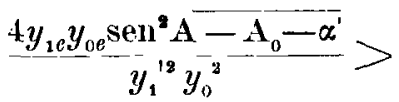

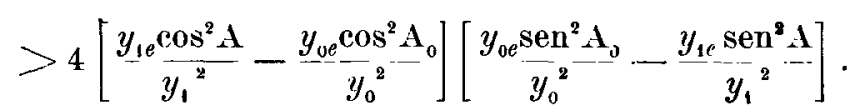

Se i due apparecchi differissero soltanto per la presenza dell'angolo $\alpha$ ' nel T. D., $\left(g_{1}^{\prime}+j b_{1}^{\prime}=g_{0}+j b_{0}\right)$, allora la disuguaglianza sarebbe francamente invertita (poichè si arrobbe $y_{1 e}=y_{v e}, \alpha_{1 e}=\alpha_{0 e}, \mathbf{A}-\mathbf{A}_{0}=\alpha^{\prime}$ ) e $I^{2}$ sarebbe immaginario. Ma, per poco che le ammettenze sieno differenti $\left(y_{1} \geqslant y_{n}\right.$ senza escludere $\alpha_{1}^{\prime}=\alpha_{0}$ ), la disuguaglianza, come è scritta, è verificata qualunque sia $y_{e}>0$. Vi sono adunque per svariate $\mathbf{Y}_{1}^{\prime}, \mathbf{Y}_{0}, \mathbf{Y}_{e}$, coppie di valori reali e positivi per $\mathbf{P}^{\mathbf{2}}$.

Di tutti questi, il più piccolo è $y_{1 e} / y_{e}$. Se difatti si introduca nella $\left(56^{\prime}\right)$ la condizione (54), sostituendovi

$$
\text { (54 bis) } \frac{y_{1}^{2} y_{0 e}}{y_{0}^{2} y_{1 e}}=\frac{4 \cos ^{2} \mathrm{~A}\left(\cos ^{2} \mathrm{~A}-\mathrm{A}_{0}-\bar{\alpha}-\cos ^{2} \mathrm{~A}_{0}\right)}{\operatorname{sen}^{2} 2 \mathrm{~A}_{0}},
$$

il discriminante diviene un quadrato perfetto,

$$
\left[y_{0}^{2} y_{4 e} \frac{\operatorname{sen} 2 A}{\operatorname{sen} 2 A_{0}} \operatorname{sen} 2\left(A-A_{0}-\alpha^{\prime}\right)\right]^{2}
$$


e l'espressione delle radici:

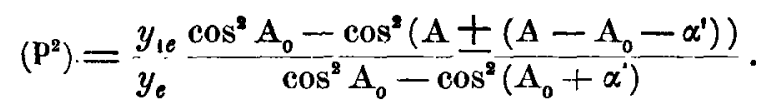

Al segno inferiore corrisponde $\mathrm{F}^{2}=y_{\mathrm{t} e} / y_{e}$; al segno superiore, un $\mathrm{P}^{2}$ negativo, da rigettarsi.

Orbene, quando la corrente differenziale si annulli, l'espressione della corrente di linea

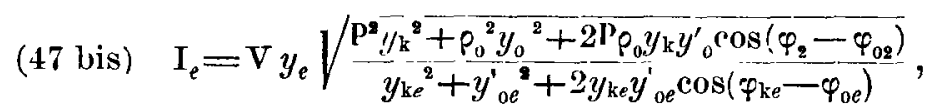

ove, mercè le (50), i rapporti dei termini corrispondenti, sopra e sotto, sono divenuti fra loro uguali, si riduce a :

$$
\left(\mathrm{I}_{e}\right)=\mathrm{V} y_{e} \frac{\mathrm{P} y_{\mathrm{k}}}{y_{\mathrm{k} e}}=\frac{2 \mathrm{~V} \mathrm{P} y_{1}^{\prime} y_{e}}{\sqrt{y_{1 e}{ }^{2}+\mathrm{P}^{\mathrm{s}}} \cdot \frac{y_{e}{ }^{2}+2 \mathrm{p}^{2}}{y_{e} y_{1 e} \cos 2 \mathrm{~A}}} .
$$

Il gradiente iniziale di questa corrente è $2 \mathrm{~V} y^{\prime} \cdot y_{e} \mid y_{1 e}$.

Con la soluzione (53), nella (57) si riproduce il primo membro della (54), e cioè :

$$
\begin{aligned}
& \left(\mathbf{I}_{e}\right)=\mathrm{V} y_{e} \frac{y_{i}^{\prime}}{\sqrt{y_{e} y_{i e}} \cos \mathrm{A}}=
\end{aligned}
$$

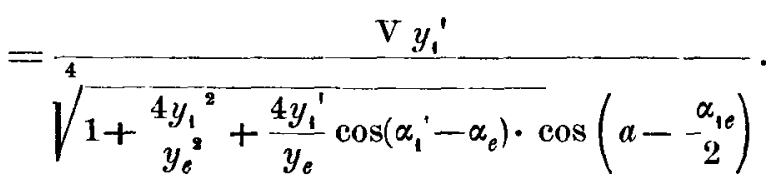

Questo valore della corrente di linea è un massimo, quello della (57); tanto più elevato e tanto più presto raggiunto, quanto più piccola sia la grandezza $y_{1 e} / y_{e},\left(\alpha_{1}^{\prime}>\alpha_{e}, 2 y_{1}^{\prime} \supseteq y_{e}\right)$. In sostanza, tanto il gradiente iniziale come il gradiente medio della funzione (57), sono proporzionati alla grandezza $y_{e}^{\prime \prime ! 10}$.

Non abbiamo considerato, nel T. D., l' esistenza del!a condizione (21*), volendo conservare al problema tutta la sua generalità; cosicchè esso fornisce le formole anche 
per due trasformatori statici $\left(\alpha^{\prime}=0\right)$ e in genere per due circuiti qualunque, sedi di forze elettromotrici alternate sincrone, lavoranti in parallelo sopra una linea $d$ ' utilizzazione.

Concluderemo, notando ancora l'espressione generale della corrente primaria $\mathrm{I}_{p}=\mathrm{V} y_{p}$, nella quale (ossia nella anmettenza complessiva $y_{p}$ ) si compendiano formalmente tutti gli effetti attivi e reattivi della coppia di trasformatori e della linea secondaria.

L'espressione simbolica della corrente primaria, quale si ricava dalle $(37)$ e $(38)$,

$$
\mathbf{J}_{p}=\mathbf{V} \frac{\mathbf{Y}_{0}^{\prime}\left(\mathbf{Y}_{0}+\mathbf{Y}_{e}\right)+\mathbf{Y}_{k}\left(\mathbf{Y}_{1}^{\prime}+\mathbf{Y}_{e}\right)+2 \mathbf{Y}_{0}^{\prime} \mathbf{Y}_{k}\left(\mathbf{1}+\mu_{0} \mathbf{Y}_{0} \cdot \mathbf{K} \mathbf{Y}^{\prime} \mathbf{Y}_{1}^{\prime}\right)}{\mathbf{Y}^{\prime}{ }^{\prime}+\mathbf{Y}_{k}+\mathbf{Y}_{e}} \mathbf{J}_{p}, \mathbf{V}, \mathbf{Y},
$$

dà per l'ammettenza complessiva:

$$
y_{p}==\frac{2 y_{0}^{\prime} y_{k}}{y_{o k e}} \times
$$

$\sqrt{\left(\frac{y_{e 0}}{2 y_{k}}\right)^{3}+\left(\begin{array}{c}y_{e i} \\ 2 y_{0}^{\prime}\end{array}\right)^{2}+\frac{y_{e 0} y_{e 1}}{2 y_{0}^{\prime} y_{k}} \cos \overline{\mathrm{A}_{e 0}-\mathrm{A}_{e l}}+2 \Theta\left(\begin{array}{l}y_{e 0} \cos \mathrm{A}_{e 0}+y_{e 2} \\ 2 y_{k}\end{array} \cos \mathrm{A}_{e 1}\right)+\theta^{2}}$

Jve si è posto :

$$
\begin{aligned}
& y_{e 0}{ }^{2}=y_{0}^{2}+y_{e}{ }^{2}+2 y_{0} y_{e} \cos \overline{\alpha_{0}-\alpha_{e}} \quad, \quad y_{e 1}{ }^{2}=y_{1}^{\prime 2}+y_{e}{ }^{2}+2 y_{1}^{\prime} y_{e} \cos \overline{\alpha_{1}^{\prime}-\alpha_{e}} \\
& \operatorname{tg} \alpha_{e 0}=\left(b_{e}+b_{0}\right) /\left(g_{e}+g_{0}\right) \quad, \quad \operatorname{tg} \alpha_{e 1}=\left(b_{e}+b_{1}^{\prime}\right) /\left(g_{e}+g_{1}^{\prime}\right) ; \\
& \mathrm{A}_{60}=\theta+\alpha_{k}-\alpha_{e 0} \quad, \quad \mathrm{~A}_{e 1}=\theta+\alpha_{0}{ }^{\prime}-\alpha_{e 1} ; \\
& \theta^{2}=1+\rho_{0}^{2} P^{2}+2 \rho_{0} P \cos \overline{a+\alpha_{0}}, \quad \operatorname{tg} \theta=\frac{\rho_{0} P \operatorname{sen} \bar{a}+\bar{\alpha}_{0}}{1+\rho_{0} P \cos \overline{a+\alpha_{0}}} .
\end{aligned}
$$

La (58), che può anche analizzarsi sott'altre forme, offre tutti gli elementi per una deduzione e nna discussione generale dei fatti particolarmente gia visti. Ci limitereno qui a notare che il primo trinomio sotto radice, a sinistra, è indipendente dal verso della velocità nel $T$. D., mentre ne dipendono gli ultimi due termini a destra $\left(p_{0} P \gtrless 0\right)$. 


\section{15. - Kiepilogo. - Il T. D. come relais telefonico.}

In quanto precede si è dimostrato che il T. D. funziona come uno speciale trasformatore nel quale il coefficiente d'induzione mutua cresca proporzioualmente con la velocità di rotore, - le sue eqaazioni avendo la stessa forma di quelle di un trasformatore statico ove si sostituisca il « fattore d'induzione » con una grandezza complessa proporzionale alla velocita di rotore; il modulo di questa rappresenta allora ciò che può chiamarsi il « fattore d'induzione dinamico ». rrendendo quindi per variabile questo fattore, cioè la velo(ità di rotazione, ne consegue, per un circuito secondario invariabile, che: a corrente primaria costante, la corrente secondaria cresce proporzionalmente alla velocità; a tensione primaria costante, la corrente secondaria cresce fino ad Iu massimo, per diminuire in seguito lentamente. L' anmentare del fattore d'induzione con la velocità corrisponde ad una trasformazione continuamente crescente di energia meccanica in energia elettrica dello stesso periodo di quella che eccita il primario.

Sotto altro punto di vista, si può dire che l'apparecchio. caricato di una data linea secondaria, offre alla tensione primaria applicata una ammettenza, funzione della velocirà del rotore, la quale ra erescendo dall'origine fino ad un massimo, con una legge espressa dalla radice quadrata di un polinomio nei termini quadratici della velocità. Al primo ramo crescente dell'ammettenza, corrispondono una corrente primaria ed una corrente secondaria crescenti verso dei massimi particolari, ed una amplificazione proporzionata della energia elettrica applicata al primario.

Il valore massimo che l'ammettenza può raggiungere, per un dato apparecchio, dipende dalle caratteristiche della linea secondaria. Sarà possibile calcolare un apparecchio per una linea determinata, o viceversa uua linea per un dato apparecchio, per modo che il gradiente iniziale dell'ammettenza, 
o quello medio intorno ad una certa velocità di regime, sia il più grande possibile.

Queste ed altre proprietà del T. D. vennero dimostrate per una determinata frequenza di corrente sinoidale, con procedimenti teorici non aventi altra pretesa che la ricerca di nozioni d'orientamento d'ordine generale e di prima approssimazione. Ridotte le equazioni della macchina a quelle di un trasformatore statico, sono note o prevedibili le particolari modificazioni da introdure nelle deduzioni teoriche per riguardo alle proprietà isteretiche degli inevitabili nuclei magnetici e alle particolarità di struttura che caratterizzano l'apparecchio in confronto a quelle ormai classiche delle macchine per forti correnti e basse frequenze.

Per l'applicazione del 'T. $D$. come relais telefonico, rimarrebbe ad analizzare il suo funziouamento con riguardo alle proprietà della linea della corrente telefonica. Dobbiamo qui limitarei alle constatazioni di fatto più essenziali.

Sebbere le formule teoriche mostrino complicate dipendenze delle leggi dell'apparecchio dalla frequenza, l'esperienza ha però mostrato che la differenza di trattamento che il T. D. offre ai vari periodi acustici è pochissimo avvertita dall'orecchio al telefono secondario, quando si ecciti il primario con una corrente microfonici modulata dal discorso o da altri suoni qualnnque, piò o weno musicali. L'apparechio conserva cioè sostanzialmente alla voce secondaria lo stesso grado di chiarezan (articolazione e tempera) che un telefono constata nella corrente primaria. Ise due vori vanno sensibilmente al passo. Pure, nella corrente riceruta, indotta e trasformata più volte di seguito, le molteplici componenti semplici hanno certamente acquistato variazioni di ampiezze e di fasi, in funzione delle singole frequenze e delle costanti di tempo dei circuiti indotti.

Se ne può concludere, da un lato, che le differenze d'amplificazione e di sfasamento per le diverse frequenze non sono gran cosa ver riguardo all' intervallo delle frequenze parlate 
o cantate; e d'altro lato, che l'orecchio interviene, qui come in tanti altri casi, con le sue virtù di abitudine integratrice, per rimettere virtualmente al passo le componenti spostate di pochi quarti di periodo durante il multiforme processo induttivo.

Si potrebbe giustificare, almeno parzialmente, questo risultato pratico, osservando che il rapporto di trasformazione fondamentale delle forze elettromotrici, $\mathrm{P},\left(21^{\prime}\right)$, è una funzione tanto più debole della frequenza, quanto più piccola sia la resistenza ohmica nel rotore e quanto più stretto sia il concatenamento magnetico fra statore e rotore.

§ 16. - Per la simmetria di principio e costruttiva deI T. D., il relais funziona indifferentemente nei due sensi, senza necessitare alcuna manovra per parte dei due corrispondenti e senza richiedere complicazioni di circuiti accessori (repeater circuits), che per altri relais hanno invece importanza fondamentale. I due avvolgimenti di statore avendo identici eJementi d' impedenza, il relais potrà stazionare verso la mezzeria della linea totale di trasmissione. Però, nulla obbliga in pratica a mantenere questa condizione costruttiva, che ci ha servito unicamente a semplificare e la teoria e la prima costruzione. È chiaro che l'apparecchio può possedere numeri differenti di spire nei due avvolgimenti, ad esempio come un trasformatore statico elevatore di tensione, per dare sulla linea secondaria una tensione soprielevata rispetto a quella primaria, indipendentemente dall' effetto amplificatore dinamico; in tal caso, la linea secondaria sarà una lunga linea, il primario del T. D. essendo alimentato dalla corrente microfonica di partenza. Se la lunga linea sia sufficientemente sprovvista o corretta da deformazioni, la invertibilità del relais resterà sempre valida.

Non si trasmette attraverso il T. D. se il rotore non sia in movimento $\theta$ se non abbia anzi raggiunto una certa velocità; poichè esso deve creare da zero la corrente telefonica 
nel secondario. Si può però ovviare fondamentalmente a questo difetto, che d'altronde è comune a tutti i sistemi di relais, utilizzando una proprietà importante del T. D., la quale permette di sommare in qualche modo la corrente, o la tensione, secondaria con la corrente, o la tensione, primaria. Infatti, le differenze di fase fra tensione secondaria e primaria, come fra corrente secondaria e primaria, sono indipendenti dalla velocità, djpendono cioè soltanto dagli elementi d' impedenza interni del T. D. e da quelli di linea.

Inoltre, queste differenze di fase sono dell'ordine di $2 \pi$, ossia, i vettori reometrici nel secondario dànno generalmente forti componenti in fase con i vettori corrispondenti nel primario. Ed esiste una condizione costruttiva limite, la quale riconduce la forza elettromotrice secondaria esattamente in fase con la tensione primaria : è la condizione di optimum per tutte le applicazioni dell'apparecchio.

Anche queste proprietà del T. D. furono dimostrate per uni determinata frequenza. Nel problema telefonico, bisognerà ridursi, come d'ordinario, a calcolare o a regolare le cose per la frequenza media di $\mathbf{8 0 0}$ periodi, o altra. Al resto, l'orecchio provvederà.

Furono studiate per questo scopo varie disposizioni.

Il T. D. pıò dapprima venire utilizzato come autotrasformatore, eccitando con la corrente primaria uno degli arvolgimenti, congiunto in serie con l'altro, e facendo partire la linea secondaria delle estremitit di questa serie. A rotore fermo, passa sulla linea secondaria buosa parte della corrente primaria; il movimento del rotore produce poi un effetto amplificatore, sommando alla tensione primaria la forza elettromotrice secondaria. Nel caso optimum, la tensione disponibile sulla linea diviene $V(1+P)$, ove $P$ è proporzionale alla velocità.

Si possono poi collegare le due linee primaria e secondaria con un trasformatore statico e un T. D. lavoranti di conserva. Il T. S. manda sulla linea secondaria la sua corrente 
di induzione e il T. 1). interviene per elevare la energia trasmiessa.

I due apparecchi possono connettersi in serie appure in parallelo fra le due linee. Anche per questi sistemi composti, l' insieme costituito dai due apparecchi S. e D. e dalla linea secondaria, equivale ad una ammettenza complessiva offerta alla tensione primaria, che è funzione della velocità del rotore; in ogni caso è possibile fissare le condizioni interne ed esterne che rendono questa ammettenza funzione crescente della velocità fin dall'origine.

Per una data connessione dei due apparecchi, la legge secondo la quale nel primo caso si sommano le tensioni e nel secondo caso si sommano le correnti indotte secondarie, cambja in generale quando si inverta il senso di rotazione nel T. D. Mentre in quest'ultimo, quando funziona da solo, le componenti della impedenza dipendono soltanto dal quadrato della relocità, se esista invece esternamente una induzione mutua costante fra i due circuiti, procurata dal T. S. asso ciato, nella espressione della impedenza risultante compaiono anche dei termini proporzionali a potenze dispari della velo cita e del fattore $d$ 'induzione esterno, $i$ quali possono essere positivi o negativi. Per certe condizioni negli apparecchi e nella linea, tali termini possono però divenire trascurabili, e la legge dell' impedenza torna allora ad essere indipendente dal verso della velocità. Altre condizioni poi concorrono ad aumentare il gradiente iniziale o il gradiente medio della curva d'ammettenza, come ad es. quella che gli avvolgimenti corrispondenti nel T. S. e nel T. D. abbiamo costanti di tempo dello stesso ordine.

Per approssimarsi a tali condizioni bisogua in pratica procedere per tentativi. Conviene che il T. S. sia una bobina d'induzione a riluttanza variabile, mediante modificazione del nucleo o altrimenti. ì poi possibile variare o correggere lo costanti di tenupo, mediante condensatori proporzionati, in terivazione (fig. 21) se i due apparecchi sono in serie, op- 
pure in serie (fig. 22) se i due apparecchi sieno in parallelo. Si regolano gli elementi dei circuiti cos̀ combinati per la

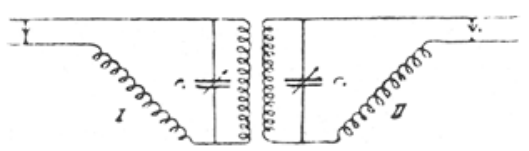

Fig. 21.

frequenza telefonica media di 800 periodi, davanti alla scala del reometro Duddell, inserito nella linea secondaria o nel

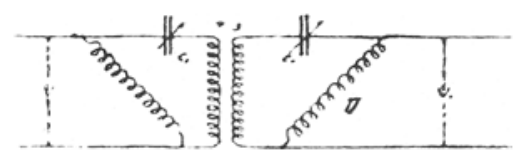

Fig. 22.

circuito che ne fa le veci, fino ad ottenere una legge d'amplificazione col gradiente medio più elevato, in funzione della velocità, o per la velocità massima di esercizio.

Il diagramma in (fig. 23) mostra alcune curve reometriche così rilevate, a 800 periodi, nel secondario del T. D. a nove dischi di rame, associato con una bobina d' induzione. Questa bobina, che fu la stessa nelle due serie d'esperienze, ‘ui sotto acennate, è rettilinea (lunghezza $16 \mathrm{~cm}$., diametro interno dell'avvolgimento $1,5 \mathrm{~cm}$., esterno $4 \mathrm{~cm}$.), è divisa in 8 sezioni adiacenti e isolate fra loro, ciascuna avvolta a 800 spire con due fili di rame paralleli di $0,2 \mathrm{~mm}$.

La resistenza totale di ciascuno dei due avvolgimenti ̀̀ di $17,5 \times 8=140 \mathrm{Ohm}$. Vari nuclei scorrevoli, in filo di ferro sottilissimo, introdotti nel tubo della bobina, possono far variare la sua impedenza effettiva (a 800 periodi) da circa $500 \mathrm{Ohm}$ fin verso $2000 \mathrm{Ohm}$. Gli avvolgimenti del T. D. (composti di $24 \times 8=192$ bobine) hanno una resistenza effettiva di $1480 \mathrm{Ohm}$ ed una impedenza effettiva di $1948 \mathrm{Ohm}$, 
alla stessa frequenza. Il circuito secondario, lo stesso nelle due serie di esperienze, è rappresentato da una semplice resisteuza ohmica di $2 \times 11110$ Ohm.

La tensione a 800 periodi applicata di due primari, proveniva dal piccolo alternatore $S$. $-H$. a ruota dentata, fortemente eccitato e frenato, con 1 'intermediario di un trasformatorino chituso, avente due avvolgimenti secondari a filo grosso, l'uno dei quali, cortocircuitato sopra un amperometro termico testimone, assorbiva buona parte del carico totale $e$ concorreva a mantener costante la tensione sul secondo avvolgimento, alimentante il sistema dei due primari.

La corrente secondaria, caricata sulla resistenza di 22000 Ohm, veniva misurata dal Duddell, col sussidio di una disposizione potenziometrica a corrente continua, analoga a quellá già descritta al §. 10

Le curve 1 e 2 del diagramma si riferiscono alla corrente secondaria data dal T. D. e dal T. S. in serie, secondo lo schema della (fig. 21). Le curve 3 e 4 son date dai due medesimi apparecchi in parallelo, come nella (fig. 22). Nelle due serie di esperienze, di carattere comparativo, si ebbe cura di mantenere costantemente ad uno stesso valore l' indicazione dell'amperometro testimone nel circuito d'alimentazione primario.

Le curve 1 e 2 rappresentano dunque l'effetto secondario col T. D. e il T.S. in serie, quando l'impedenza della bobina d' induzione che fa da $T$. S. venga corretta con i condensatori derivati $\mathrm{C}_{1}=\mathrm{C}_{2}=0,15 \mu \mathrm{F}$. Delle capacità più grandi ovvẹo più piccole, danno altre curve di gradiente medio meno elevato. Si passa dai punti della curva 1 a quelli della curva 2, semplicemente rovesciando le connessioni del primario della bobina d' induzione col primario del T. D. mediante un commutatore. Come si scorge, v'ha una velocità (3600 giri) per la quale l'amplificazione è la medesima con entrambe le connessioni. Lo stesso si otterrebbe, per una data connessione, rovesciando il verso della velocità. 
Le curve 3 e 4 rappresentano l'effetto di corrente secondario con il collegamento dei due apparechi in parallelo e un dato verso della velocità (quello più conveniente). Si ot-

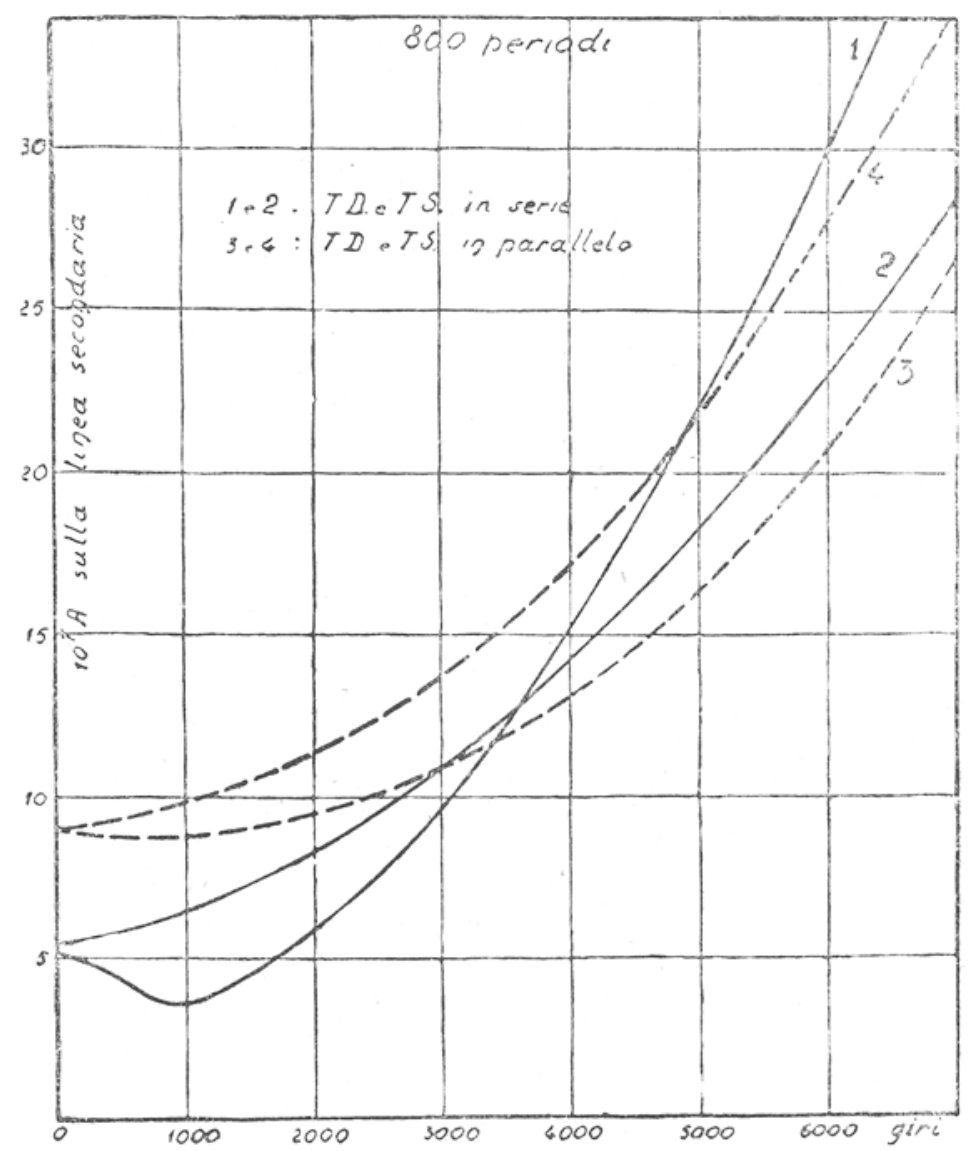

Fig. 23.

tenue la curva 3 , con $\mathrm{C}_{1}=0,15 \mu \mathrm{F}, \mathrm{C}_{1}=\infty$; la curva 4, con $\mathrm{C}_{1}=\mathrm{C}_{2}=0,15 \mu \mathrm{F}$, superiore alla prima $\mathrm{e}$ con gradiente injziale positivo mentre nella prima è negativo. Fra 7000 e e 8000 giri, queste curve acquistano poi dei flessi, per dirigersi probabilmente verso dei massimi. Applicando piccole 
capacità $(0,01 \ldots \mu \mathrm{F})$ in parallelo sulla linea, i punti di queste curve si elevano sensibilmente, capacità più noterolí $(0,1 \ldots \mu \mathrm{F})$, li abbattono.

Questi risultati di esperienze che qui abbiamo potuto riferire, illustrano concordemente la proprietà fondamentale del T. D., di possedere una ammettenza risultante la quale va crescendo inizialmente con la velocità di rotazione. La legge di questa funzione può venire notevolmente modificata, oltre che dai caratteri costruttivi dell'apparechio, dalle condizioni d' impedenza della linea d'utilizzazione. 